\title{
Nanoscale-specific bioassimilation of sulfur: Time and coating specific modulation of transcriptomic and metabolomic pathways in diseased tomato
}

\section{Yi Wang ( $\sim$ Yi.Wang@ct.gov )}

The Connecticut Agricultural Experiment Station https://orcid.org/0000-0002-2891-0386

\section{Chaoyi Deng}

The University of Texas at El Paso

\section{Sudhir Sharma}

University of Massachusetts Amherst

\section{Gilberto Navarro}

The University of Texas at El Paso

\section{Jacquelyn LaReau}

The Connecticut Agricultural Experiment Station

\section{Zhengyang Wang}

The Connecticut Agricultural Experiment Station

\section{Christian Dimkpa}

The Connecticut Agricultural Experiment Station https://orcid.org/0000-0003-2143-5452

\section{Blaire Steven}

The Connecticut Agricultural Experiment Station

\section{Zhenyu Wang}

Institute of Environmental Processes and Pollution Control, and School of Environmental and Civil Engineering, Jiangnan University

\section{Lijuan Zhao}

Nanjing University https://orcid.org/0000-0002-8481-0435

Chunqiang Li

The University of Texas at El Paso

\section{Om Dhankher}

University of Massachusetts https://orcid.org/0000-0003-0737-6783

\section{Baoshan Xing}

University of Massachusetts Amherst https://orcid.org/0000-0003-2028-1295

\section{Jorge Gardea-Torresdey}

University of Texas at El Paso

\section{Wade Elmer}

Connecticut Agricultural Experiment Station https://orcid.org/0000-0003-3308-4899 
Jason White

The Connecticut Agricultural Experiment Station https://orcid.org/0000-0001-5001-8143

\section{Article}

Keywords: sulfur nanoparticles, disease suppression, Fusarium wilt, metabolomics, nano-enabled agriculture, food security, sustainability

Posted Date: December 9th, 2021

DOI: https://doi.org/10.21203/rs.3.rs-1128848/v1

License: (c) (1) This work is licensed under a Creative Commons Attribution 4.0 International License. Read Full License 


\section{Abstract}

Nanoscale sulfur was investigated as a multi-functional agricultural amendment to simultaneously enhance crop nutrition and suppress disease damage. Pristine (nS) and stearic acid coated (cS) sulfur nanoparticles were added to soil $(0,100$, or $200 \mathrm{mg} / \mathrm{L}$ ) that was planted with tomato (Solanum lycopersicum) and infested with the Fusarium wilt pathogen. Bulk sulfur (bS), ionic sulfate (iS), and healthy controls were included. In two greenhouse experiments, measured endpoints included timedependent agronomic and photosynthetic parameters, disease severity/suppression, and a range of mechanistic biochemical and molecular endpoints, including the expression of 13 genes related to two $S$ bioassimilation pathways and pathogenesis-response, and tissue-specific metabolomic profiles. The impact of treatment on the rhizosphere bacterial microbiome was also evaluated. Disease reduced tomato biomass by up to $87 \%$, but amendment with nS and cS significantly reduced disease progress by 54 and $56 \%$, respectively, compared to the infested controls. Increased S accumulation was evident in plant roots and leaves, independent of $S$ type. Molecular analysis revealed particle size and coatingspecific impacts on the plants. For $\mathrm{nS}$ and $\mathrm{cS}$, two-photon microscopy and time-dependent gene expression data revealed a nanoscale specific elemental $S$ bioassimilation pathway within the plant tissues. These findings correlated well with detailed metabolomic profiling of plant tissues at 4, 8, and 16 $d$, which exhibited increased disease resistance and plant immunity related metabolites with nanoscale treatment. The data also demonstrate a time-sensitive physiological window whereby nanoscale stimulation of plant immunity will be effective. An analysis of the rhizosphere soil bacterial community revealed minimal impacts from $S$ soil treatments. These findings provide significant mechanistic insight into non-metal nanomaterial-based suppression of plant disease, and significantly advance efforts to develop sustainable nano-enabled agricultural strategies to increase food production.

\section{Introduction}

Although the Green Revolution led to significant increases in agricultural output through agrochemical use and irrigation, many current practices are not sustainable ${ }^{1-3}$. For example, the efficiency of agrochemical delivery is quite low, often no more than $30 \%{ }^{4}$. This results in growers overapplying pesticides and fertilizers to achieve the acceptable yield, but leads to environmental damage ${ }^{5,6}$. In addition, agricultural production will have to increase by up to $70 \%$ by 2050 to feed an expected global population of more than 9 billion people ${ }^{7-10}$. Such increases will only be possible if new technologies and approaches can be applied across the farm to fork continuum.

In spite of the widespread use of agrochemicals, plant pathogens reduce the yield of most crops by $20 \%$ and compromise product quality, often leading to $40 \%$ food loss before harvest ${ }^{1}$. There has been increasing interest in developing nanoscale materials for agricultural use, including novel crop management strategies and targeted delivery of nutrients and pesticides ${ }^{10-13}$. Recent work has demonstrated the unique potential of nanoscale micronutrients to enhance tolerance to both biotic and abiotic stressors. Dimkpa et al. demonstrated that nanoscale zinc amendments to soil not only alleviated 
drought damage but also enhanced nutrient uptake into the sorghum grain ${ }^{14}$. Foliar amendments of nanoscale copper can suppress soil-borne fungal disease damage under both greenhouse and field conditions, enhancing the growth and yield of crops, including watermelon, eggplant, tomato, and soybean ${ }^{15-18}$. Importantly, this enhanced disease tolerance is a function of modulated nutrition, with significant increases in the expression of defense and antioxidant-related genes ${ }^{18,19}$.

Although the literature on the use of nanotechnology in food production is increasing, the majority of work has focused on metal, metalloid, or metal oxide NPs. Sulfur (S) is a secondary nutrient, being a key component of essential amino acids and protein synthesis. Several S-containing amino acids are critical to glutathione (GSH) metabolism, which is central to defense against biotic and abiotic stresses ${ }^{20}$. Interestingly, as the use of high sulfur-containing fossil fuels has decreased, inputs into soils have declined, and reports of deficiencies have occurred ${ }^{21}$. As such, the application of bulk $S$ has become more common, although the efficiency of delivery and utilization is low, and excessive application can lead to soil acidification.

A limited number of studies have evaluated the antimicrobial activity of nanoscale sulfur in vitro. Suleiman et al. ${ }^{22}$ showed that nanoscale $S$ exhibited antimicrobial activity against Staphylococcus aureus. Choudhary et al. ${ }^{23}$ demonstrated that nanoscale $S$ was more effective than the bulk form at suppressing Aspergillus niger. Cao et al. ${ }^{24}$ investigated S nanoparticle (nS) seed treatment and foliar application on tomato (Solanum lycopersicum L.) infected with Fusarium oxysporum f. sp. lycopersici. Importantly, nS treatment suppressed disease activity by $48 \%$ and increased shoot biomass by $56 \%$ when compared to untreated controls, and the authors correlated these changes with the upregulation of select pathogenesis and antioxidant-related genes. Here, we mechanistically describe the nanoscale specific accumulation of sulfur by tomato, including size and coating specific response at the level of gene expression and the metabolome over time. Prior to growth, soils were amended with 100 or $200 \mathrm{mg} / \mathrm{L} \mathrm{soil}$ of nanoscale sulfur that was or was not coated with stearic acid. Measured endpoints include disease progress, biomass, elemental/nutritional analysis, time dependent expression of disease- and stressrelated genes, LC-MS/MS based-metabolomic analysis, and an evaluation of the rhizosphere microbiome by 16 sRNA sequencing. These findings provide a detailed mechanistic understanding of nanoscale sulfur use as a soil amendment for sustainable nano-enabled agriculture.

\section{Methodology}

\section{Characterization of nanoscale S}

Pristine (99.99\%) and surface coated (1-2wt\% stearic acid) sulfur nanoparticles were obtained from US Research Nanomaterials, Inc., (Houston, TX). Both pristine sulfur nanoparticles (nS) and stearic acidcoated sulfur nanoparticles (cS) were characterized by X-ray diffraction analysis (XRD, Panalytical Empyrean 2, Malvern Panalytical Ltd., Malvern, UK). The size and morphology of pristine and surface coated nS were observed using scanning electron microscopy with energy dispersive X-ray spectroscopy (SEM-EDS) (Hitachi S-4800, Hitachi Ltd., Tokyo, Japan) and transmission electron microscopy (TEM) 
(HT7800, Hitachi Ltd., Tokyo, Japan). For TEM analysis, suspensions in acetone of both nanoscale sulfur types were sonicated for $15 \mathrm{~min}$ in cold water. Aliquots were then added dropwise to a Cu grid with lacey carbon film and were allowed to air dry. A Malvern Zetasizer Nano ZS-90 following a laser doppler electrophoresis procedure was used to measure hydrodynamic diameter and zeta potential of the $S$ based compounds at $200 \mathrm{mg} / \mathrm{L}$.

\section{S dissolution and soil bioavailable nutritional elements}

The dissolution of sulfate from different sulfur types $(200 \mathrm{mg} / \mathrm{L})$ was measured in batch reactors. There were five replicates for each treatment; each replicate was sonicated on ice for 2 minutes at $120 \mathrm{~W}$ (Fisherbrand ${ }^{\mathrm{TM}}$ Model 120 Sonic Dismembrator) and kept in the dark to avoid photodegradation. Five ml samples were taken at $4 \mathrm{~h}, 1$ day, $4 \mathrm{~d}, 8 \mathrm{~d}$, and $16 \mathrm{~d}$ and were centrifuged (Eppendorf ${ }^{\mathrm{TM}}$ Centrifuge 5810R) at $12,000 \mathrm{rpm}$ for 30 minutes at $4^{\circ} \mathrm{C}$. The supernatant was then transferred into clean vessels, acidified with $\mathrm{HNO}_{3}$, and analyzed using inductively coupled plasma- optical emission spectroscopy (ICP-OES) (see below) for $\mathrm{S}$ content. To determine the bioavailability of soil nutrients, $2.0 \mathrm{~g}$ aliquots of air-dried soil were extracted by two procedures modified according to a previous study ${ }^{25}$. First was a DTPA method using with $30 \mathrm{~mL}$ mixture of $0.01 \mathrm{M} \mathrm{CaCl}_{2}, 0.005 \mathrm{M}$ DTPA and $0.1 \mathrm{M}$ triethanolamine (TEA) ( $\left.\mathrm{pH}=7.6\right)$. The second procedure used $30 \mathrm{~mL}$ of $0.01 \mathrm{M} \mathrm{CaCl}_{2}(\mathrm{pH}=5)$. DI water $(30 \mathrm{~mL})$ was also used as a third extracting solution. All extractions were conducted on a reciprocal shaker for 2 hours at $180 \mathrm{rpm}$ to facilitate mixing. The samples were then centrifuged for $10 \mathrm{~min}$ at 12,000 rpm, and the supernatants were passed through a 0.2 um PTFE filter. The $\mathrm{pH}$ and electrical conductivity (EC) of DI extracts were measured to estimate the soil pore water pH and soil salinity (Fig. S18 and Table S3). All supernatants were analyzed by ICP-OES as described below.

\section{Greenhouse experiments}

Seeds of Solanum lycopersicum L. cv Bonnie Best were obtained from Harris Seed Co., Rochester NY; this is a common heirloom variety which is susceptible to Fusarium wilt. Seeds were germinated in Pro-Mix BX potting soil (Premier Hort Tech, Quakertown, PA) (with a background S level of $218.4 \pm 5.5 \mathrm{mg} / \mathrm{L}$ ) in 36 cell $(5.66 \times 4.93 \times 5.66 \mathrm{~cm})$ plastic liners and grown for four weeks. Twenty-five $\mathrm{ml}$ of a low sulfur $(0.035 \%)$ soluble fertilizer (Premium Water Soluble Plant Food, 24-13-17, NPK, Proven Winners, USA) was applied at the end of the third week, and was given once per week after seedling transplanting. Seedlings of uniform size with three to four leaves were selected for experiments. F. oxysporum f. sp. lycopersici inoculum was prepared according to our previous studies ${ }^{15,17,26-28}$. Briefly, autoclaved millet seeds were seeded with agar plugs colonized by F. oxysporum at $22-25^{\circ} \mathrm{C}$ for two weeks. The millet was dried, ground, and passed through a $1 \mathrm{~mm}$ sieve. The millet was added at $0.75 \mathrm{~g} /$ liter potting soil and 
thoroughly mixed to yield approximately $7.5 \times 10^{4}$ Fusarium colonies per g potting mix as determined by serial dilution onto $25 \%$ potato-dextrose-agar (Difco, Corpus Christi, TX) ${ }^{15}$.

The efficacy of $\mathrm{nS}$ and $\mathrm{cS}$ at suppressing Fusarium infection on tomatoes was evaluated and compared with corresponding bulk form. To assess the effect of surface coating/charge, the responses to $\mathrm{nS}$ and cS were directly compared. All S based materials were applied by a soil amendment.

Suspensions/solutions of each S-based compound were prepared in $25 \mathrm{~mL}$ deionized water (DI), followed by sonication for 2 min using a probe sonicator to provide a stable dispersion. The prepared solutions were then mixed into the potting soil prepared above to achieve final concentrations of $200 \mathrm{mg}$ $\mathrm{S} / \mathrm{L}$ soil, and manually mixed for $20 \mathrm{~min}$. Twenty-five $\mathrm{mL} \mathrm{DI}$ water was added to potting mix to serve as an untreated control. Uniform 4-week-old tomato seedlings were transplanted into these soils (one per pot) and were placed in a greenhouse before harvest under the following conditions: $25-28{ }^{\circ} \mathrm{C}$ day $/ 20-22$ ${ }^{\circ} \mathrm{C}$ night, relative humidity of $60-70 \%$, under high intensity sodium vapor lights set for 12 -hour photoperiods. The time-dependent effects of treatment were evaluated; here, three plants were harvested at 4,8 , and 16 days after transplanting. In a second experiment of similar design, an additional concentration of $100 \mathrm{mg} \mathrm{S} / \mathrm{L}$ soil and an additional treatment of ionic sodium sulfate $\left(\mathrm{Na}_{2} \mathrm{SO}_{4}\right)$ was added to address the effect of dose and a non-nano ionic $S$ form. There were 10 replicates, and plants were harvested at 35 days after transplanting. Photosynthetic parameters including relative chlorophyll content, leaf thickness, and linear electron flow (LEF) were measured using a portable PhotosynQ (PHOTOSYNQ INC., USA) at $30 \mathrm{~d}$ after the transplanting. For both greenhouse experiments, root stem and leaf tissue were separately weighed, washed in tap water, rinsed with $0.01 \%$ nitric acid, rinsed again with $\mathrm{DI}$, frozen in liquid nitrogen, and then stored at $-80^{\circ} \mathrm{C}$ until further analysis.

\section{Disease progress}

Disease severity of $F$. oxysporum infected tomato across all treatments was determined every other day on a scale of 1 to $5: 1$ = asymptomatic, 2 = slightly stunted and/chlorotic, 3 = partially stunted or wilted, 4 $=$ serious stunted or wilted, and $5=$ dead or near death. Disease progress was plotted over time, and the area-under-the-disease-progress curve (AUDPC) was calculated using the trapezoid rule where: AUDPC = $\sum\left(Y_{i}+Y_{i}\right) / 2 \times\left(t_{i+1}-t_{i}\right)$, where $Y_{i}=$ disease rating at time $t_{i}$. The pathogen was re-isolated from damaged stem tissue to confirm its association with the disease.

\section{Elemental Analysis}

Harvested tissues were analyzed by ICP-OES (iCAP 6500, Thermo Fisher Scientific, Waltham, MA) to measure $S$ accumulation, as well as secondary and macronutrients ( $\mathrm{Ca}, \mathrm{Mg}, \mathrm{P}$, and $\mathrm{K}$ ) and micronutrients $(\mathrm{B}, \mathrm{Cu}, \mathrm{Fe}, \mathrm{Mn}, \mathrm{Mo}, \mathrm{Zn})$. Briefly, $150 \mathrm{mg}$ of dry ground tissue was weighed, and digested in $5 \mathrm{~mL}$ plasma pure $\mathrm{HNO}_{3}$ on a hot block at $115^{\circ} \mathrm{C}$ for $45 \mathrm{~min}$. The digesta was amended with $1 \mathrm{~mL}$ of $\mathrm{H}_{2} \mathrm{O}_{2}$ and further 
digested for $20 \mathrm{~min}$ at $115^{\circ} \mathrm{C}$. The digests were then diluted to $50 \mathrm{~mL}$ with DI. To validate the measurements, samples consisting of a blank (no plant tissues), pure nanoscale and bulk $\mathrm{S}$ powder, and standard reference materials (NIST-SRF 1570a and 1547, Metuchen, NJ) were prepared, digested, and analyzed by the same procedure. Yttrium $(\mathrm{Y})$ was used as an internal standard, and a continuing calibration verification sample was evaluated every 15 samples.

\section{RNA Isolation and RT-qPCR}

For gene expression analysis, tissue samples that had been frozen in liquid $\mathrm{N}_{2}$ were pulverized with a mortar and pestle. Total RNA was extracted using the PureLink ${ }^{\text {TM }}$ Plant RNA Reagent (Invitrogen ${ }^{\text {TM }}$ ) according to the manufacturer's instructions and was quantified using a NanoDrop spectrophotometer (Thermo Scientific). RNA quality and integrity were confirmed by the 260/280 Abs ratio. RT-qPCR analysis was conducted as described previously ${ }^{29}$; RNA was exposed to RNase-free DNAse I (Roche) at $37{ }^{\circ} \mathrm{C}$, and then cDNA was synthesized by the High-Capacity cDNA Reverse Transcription Kit with RNase Inhibitor (ThermoFisher Scientific). The final concentration of cDNA was $5 \mathrm{ng} / \mathrm{mL}$. Quantitative PCR (qPCR) was performed in a StepOne ${ }^{\mathrm{TM}}$ Real-Time PCR system. PCR reaction tubes were amended with $\mathrm{iQ}^{\mathrm{TM}} \mathrm{SYBR}{ }^{\circledR}$ Green Supermix (Biorad) and primers at a $20 \mu \mathrm{L}$ final volume. The PCR conditions were: 1 cycle at $95^{\circ} \mathrm{C}$ for $5 \mathrm{~min} ; 40$ cycles at $95^{\circ} \mathrm{C}$ for $15 \mathrm{~s}, 55^{\circ} \mathrm{C}$ for $25 \mathrm{~s}$, and $72^{\circ} \mathrm{C} 20 \mathrm{~s}$, including a final melting curve program at $60{ }^{\circ} \mathrm{C}+0.3$ for $20 \mathrm{~s}$. The selected genes and the corresponding primers are described in Table S22. The PCR amplification efficiency was calculated with different primers with a range between 90 and $110 \%$. Gene expression analysis was done following the $2^{\Delta \mathrm{Ct}}$ method.

\section{Two-photon microscopy analysis}

Tomato root and leaf samples were cut with a CryoMicrotome (Triangle Biomedical Sciences, Durham, $\mathrm{NC}$ ) at $-20^{\circ} \mathrm{C}$. The resulting sections were mounted on glass substrates and viewed under a waterimmersion objective lens (Olympus LUM Plan FLN) ${ }^{30,31}$ using a mode-locked Ti: Sapphire laser (SpectraPhysics, Mai-Tai HP) as the light source. Two-photon excitation was achieved at a wavelength of $720 \mathrm{~nm}$ and $150 \mathrm{~mW}$. The pulse duration and the repetition rate were $100 \mathrm{fs}$ width and $80 \mathrm{MHz}$, respectively. The blue and green/red fluorescence signals were deflected with a long-pass dichroic mirror at $665 \mathrm{~nm}$, and split through a long-pass dichroic beam splitter, transmitted through band-pass filters of 417-477 nm, 500-550 nm, and 570-616 nm, respectively, and detected by a photomultiplier tube at $2.10 \mathrm{~V}, 2.20 \mathrm{~V}$, and $0.9 \mathrm{~V}$, respectively. The outputs were fed into red/green/blue channels by a frame grabber. The speed of imaging was 30 frames/s, and a static image was obtained as an average of 100 frames $^{32}$.

\section{Metabolomic analysis and data processing}


Fresh leaf tissues collected at harvest were immediately frozen in liquid nitrogen for $20 \mathrm{~s}$ for complete metabolic inactivation and stored at $-80^{\circ} \mathrm{C}^{33}$. They were then freeze-dried for $6 \mathrm{~h}$ using a lyophilizer. Dry samples ( $8 \mathrm{mg}$ ) were extracted in $2 \mathrm{ml}$ of cold $80 \%$ LC-MS-grade methanol containing $100 \mathrm{ng} / \mathrm{mL}$ of metalaxyl as the internal standard. Untreated healthy tomato leaves were used as a control. Mixtures were consecutively vortexed and sonicated in ice cold water bath for $20 \mathrm{~min}$. The extracts were centrifuged for $15 \mathrm{~min}$ at 14,000 rpm, and the supernatants were used for detection using a Waters Acquity ultra-high performance liquid chromatography (UPLC) system directly coupled to a Waters Synapt G2Si HDMS high resolution mass spectrometer. For all experiments, $10 \mu \mathrm{L}$ of metabolite extract was directly loaded onto a Waters Acquity UPLC HSS T3 analytical column with $100 \AA$ A pore size, $1.8 \mu \mathrm{m}$ particle diameter, $2.1 \mathrm{~mm} \times 150 \mathrm{~mm}$, that was held at $40^{\circ} \mathrm{C}$. A reversed phase binary gradient using Fisher Optima LC/MS grade solvents (Solvent A: $0.1 \%$ formic acid in water, Solvent B: $0.1 \%$ formic acid in acetonitrile) was used to elute metabolites directly into the mass spectrometer. The gradient included a $0.300 \mathrm{~mL} / \mathrm{min}$ flow rate and the following conditions for elution: $0.2 \mathrm{~min}$ initial hold at $1 \%$ Solvent $\mathrm{B}$, a linear ramp to $60 \%$ Solvent B over 4.8 min, a linear ramp from $60 \%$ to $90 \%$ Solvent B over $0.5 \mathrm{~min}$, a 0.7 min hold at $90 \%$ Solvent B, a linear ramp from $90 \%$ to $1 \%$ Solvent B over 0.2 min and a re-equilibration wash at $1 \%$ Solvent $B$ for 2 min.

The Synapt G2Si mass spectrometer was kept in positive mode electrospray ionization and the following tune parameters: $+3 \mathrm{kV}$ capillary voltage, $40 \mathrm{~V}$ sampling cone voltage, 80 source offset, source temperature of $100^{\circ} \mathrm{C}$, cone gas flow of $50 \mathrm{~L} / \mathrm{h}$, desolvation gas flow of $832 \mathrm{~L} / \mathrm{h}$, and nebulizer pressure of 6.5 bar. An 8.4 min alternating full MS and $\mathrm{MS}^{\mathrm{E}}$ data-independent acquisition method was implemented using positive polarity and the "Resolution" acquisition mode. The following parameters were used for Full MS acquisition: mass range of 100 to $1,000 \mathrm{Da}, 0.2 \mathrm{sec} / \mathrm{scan}$ time, Trap fixed collision energy of $4 \mathrm{~V}$, with no additional Transfer collision energy. $\mathrm{MS}^{\mathrm{E}}$ scans were collected with the following parameters: mass range of 50 to $1,000 \mathrm{Da}, 0.2 \mathrm{sec} / \mathrm{scan}$ time, Trap collision energy ramp from 5-40 V, and no additional Transfer collision energy. A lock spray mass correction was performed in real-time using infused Leucine Enkephalin $(556.2771 \mathrm{~m} / \mathrm{z}$ ) and the following acquisition settings: a $0.2 \mathrm{sec} / \mathrm{scan}$ time, acquisition interval of $10 \mathrm{sec}, 3$ scans to average, and a mass window of $\pm 0.5 \mathrm{Da}$.

All raw data were processed and analyzed directly using Progenesis QI (v 2.4, Nonlinear Dynamics) for pick picking, feature retention time (RT) alignment, feature quantification, and metabolite identification. All raw files were imported using the "High resolution mass spectrometer" settings with profile data and positive polarity. RT alignment was achieved using the "Assess all runs in the experiment for suitability" option, which resulted in successful alignment across the dataset (scores $>87 \%$ ). Peak picking used "Automatic" for lon Sensitivity, included no RT limits, used " $0.2 \%$ of base peak" for the high energy fragment ion intensity limit, and included the following adducts: $\left(\mathrm{M}+\mathrm{H}^{+}\right)^{+},\left(\mathrm{M}+2 \mathrm{H}^{+}\right)^{2+},\left(\mathrm{M}+\mathrm{Na}^{+}\right)^{+}$, and $\left(\mathrm{M}+\mathrm{K}^{+}\right)^{+}$. Experiment-wide normalization was completed using the "Normalize to all compounds" option. The entire molecular feature list was searched against the METLIN spectral library database using a 10-ppm tolerance for precursor masses, a 20-ppm tolerance for fragment ion masses, and only "empirical" fragmentation type. Quantified feature information and METLIN-derived molecular 
identifications were exported from Progenesis QI for additional analysis. Statistical analysis and pathway analysis of the metabolites was performed using MetaboAnalyst 5.0 For multivariate analysis, $\log 10(\mathrm{x})$ transformation was performed for the normalization by sum ${ }^{34,35}$. One-way ANOVA followed by Fisher's LSD test $(p \leq 0.05)$ was performed to screen for metabolites. A supervised partial least-squaresdiscriminant analysis (PLS-DA) was applied to get a global overview of the variation of metabolic profiles. The library used in pathway enrichment and pathway topology analysis is the Arabidopsis thaliana KEGG library. The impact value threshold $>0.1$ was considered as significantly affected. The features with a VIP greater than 0.1 are regarded as responsible for separation ${ }^{36}$.

\section{Rhizosphere bacteria characterization}

Rhizosphere soils were defined as the soil tightly adhering to the root tissues and were collected as described previously ${ }^{37}$. At harvest, ten-cm sections of tomato roots were collected from each plant and shaken vigorously to remove loosely adhering soil. The root sample was then transferred to a centrifuge tube containing $25 \mathrm{ml}$ of sterile phosphate-buffered saline. The rhizosphere soil was removed from the roots by vortexing the samples for 2 min at full speed. The roots were then removed with ethanolsterilized forceps, and soils were concentrated by centrifugation at $14,000 \mathrm{rpm}$ for $10 \mathrm{~min}$ at $4^{\circ} \mathrm{C}$. The concentrated rhizosphere soils were stored at $-80^{\circ} \mathrm{C}$ until DNA extraction.

DNA was extracted from $0.25 \mathrm{~g}$ of soil from the resulting pellet using the DNeasy PowerSoil kit (Qiagen). DNA extractions were verified by gel electrophoresis in a 1\% agar gel. Bacterial 16S rRNA genes were amplified with the primer pair 515F (GTGYCAGCMGCCGCGGTAA) and 806R (GGACTACNVGGGTWTCTAAT) $^{38}$. Extracts were amplified with $10 \mu \mathrm{l}$ Platinum SuperFi II DNA polymerase (Invitrogen), which also included $7.5 \mu \mathrm{M}$ both the mPNA and pPNA peptide nucleic acid (PNA) clamps (mPNA, GGCAAGTGTTCTTCGGA; PPNA, GGCTCAACCCTGGACAG) to block the amplification of host plant mitochondria and plastid rRNA genes, respectively. PCR conditions consisted of $94^{\circ} \mathrm{C}$ for $2 \mathrm{~min}$ followed by 30 cycles of $94^{\circ} \mathrm{C}$ for $15 \mathrm{~s}, 60^{\circ} \mathrm{C}$ for $15 \mathrm{~s}, 68^{\circ} \mathrm{C}$ for $15 \mathrm{~s}$, and $4^{\circ} \mathrm{C}$ for an infinite hold. The resulting amplification products were verified by gel electrophoresis, and cleaning and normalization of individual PCR products were performed with a SequalPrep normalization plate (Invitrogen). The normalized PCR amplicons were mixed, and the quantity and quality of the DNA pool were verified using an Agilent TapeStation. The resulting 16S rRNA gene amplicons were sequenced on the Illumina iSeq 100 system employing 150 base pair chemistry.

16S rRNA gene sequences were initially processed using the mothur software package ( $\mathrm{v}$. 1.44.2 $)^{39}$. Because the $150 \mathrm{bp}$ reads were too short to generate contigs, only the forward reads were retained for analysis. Quality filtering was done and selected for sequences of at least $150 \mathrm{bp}$ in length. Chimeric sequences were identified with the VSEARCH algorithm as implemented in mothur, using the most abundant sequences as a reference for chimera detection ${ }^{40}$. All putative chimeric sequences were removed from the data sets. A total of 36,523 high-quality $16 \mathrm{~S}$ rRNA sequences were retained, with an 
average of 1,523 per sample. The 16S rRNA sequences were classified against the SILVA v138 reference database using the RDP naive Bayesian classifier as implemented in mothur, and sequences identified as

belonging to eukaryotes were removed ${ }^{41}$. The resulting sets of sequences were assigned to amplicon sequence variants, employing a $100 \%$ sequence similarity threshold. The mothur output files were imported into the microeco $\mathrm{R}$ package for descriptive and statistical analyses. Biomarkers distinguishing the different treatments were identified with linear discriminant analysis (LDA) effect size (LEfSe) method $^{42}$.

\section{Statistical analysis}

A Shapiro-Wilk's W Test of homogeneity was performed on all data before further analysis. A one-way ANOVA followed by a Tukey-Kramer multiple comparison test was conducted (SPSS, IBM Corp.) with the $S$ treatments as fixed factors. Experiment two was similarly analyzed with $S$ treatment and dose as fixed factors. A student's t-test was also used to compare the differences between specific treatments. Statistical significance was determined at $p \leq 0.05$. Data are expressed as the mean \pm standard error (SE).

\section{Results}

\section{S material Characterization}

Images from the SEM-EDS, TEM, and XRD patterns are shown in Fig. S1-3. Both uncoated and coated nanoscale $S$ showed a uniform spheroidal shape with average particle sizes of 65 and $38 \mathrm{~nm}$, respectively, and average hydrodynamic sizes of $851.2 \pm 28.7 \mathrm{~nm}$ and $982.5 \pm 86.6 \mathrm{~nm}(\mathrm{pH}=7)$, respectively (Fig. S2, S4, and Table S1). The zeta potential of nS, cS, and bS (Fig. S4 and Table S1) at pH 7 was $-23.6 \pm 0.4,-33.5 \pm 0.3$, and $-13.9 \pm 4.3$, respectively. The crystal diffraction pattern of $n S$, cS and bS from XRD analysis (Fig. S3) matched well with the orthorhombic structure of elemental sulfur $(S 8)^{43}$. The EDS analysis confirmed the elemental compositions of $n S$ and $b S$ as pure $S$, and $c S$ as a combination of $S$ and C (Fig. S1). The dissolution of all the three $S$ based compounds in DI was less than $0.4 \%$ at 15 days (Fig. S5 and Table S2).

\section{Two-photon microscopy images}

Detection of S by two-photon microscopy is both size- and coating-dependent (Fig. 1, S6, and S7). In DI suspensions at $500 \mathrm{mg} / \mathrm{L}$, much less fluorescence signal was detected from $\mathrm{nS}$ suspensions that were not sonicated, since particle aggregation significantly reduced signal intensity (Fig. 1e); after thorough sonication, the fluorescence signal from nS was clearly observed (Fig. 1k). At the same concentration, neither bS suspension with or without sonication, or ionic $S$ solutions, had a fluorescence signal (Fig. 1f, 
$\mathrm{g}, \mathrm{l}, \mathrm{m}, \mathrm{j}$, and $\mathrm{p}$ ). The finding that smaller size enables a stronger fluorescence has been reported

previously for nanoscale $\mathrm{ZnO}^{44}$. Importantly, steric acid-coated nanoscale $\mathrm{S}$ did not fluoresce (Fig. $1 \mathrm{~h}, \mathrm{i}, \mathrm{n}$, and o), likely due to the change in surface chemical composition; an ionic $\mathrm{SO}_{4}{ }^{2-}$ solution also exhibited no fluorescence. This unique fluorescence response of uncoated nanoscale $S$ will be important to understand the nanomaterial movement in the plant. The root uptake of $S$ based $(200 \mathrm{mg} / \mathrm{L})$ compounds and translocation to leaf tissues as determined by two-photon microscopy is shown in Fig. 1c, d, and S6. Representative fluorescent signals from $S$ are indicated by the arrows. These distinguishable response points are similar to the pure nanoscale S suspended in DI (Fig. 1e and k). In nS treated roots, a S fluorescent signal was evident, indicating nS accumulation; clear signals are also evident in the corresponding leaf tissue, demonstrating translocation of nanoscale S. For $\mathrm{cS}$, a weak S signal was found in CS treated roots. Given the increased S content observed by ICP-OES (below) and the lack of cS response in solution, this suggests that $\mathrm{CS}$ was accumulated and translocated through tomato either in the surface coated form or as dissolved ions. No detectable signal S was found in bS exposed roots, indicating the absence of nanoscale $S$; interestingly, significant $S$ fluorescence was shown in the corresponding leaves of bS treated plants, suggesting that active in planta transformation of bS was occurring, including likely reduction of sulfate ions to nanoscale $S$ in leaf tissues. Spatially, $S$ was observed near or in the vascular tissues and the intercellular spaces, indicating apoplastic route of uptake. In the leaf tissues, $S$ resulting from $n S$ and bS exposure was distributed similarly and primarily around the stomata, indicating a xylem-based transport pathway via the transpiration stream. Additional time resolved videos of the 3D biodistribution pattern of $S$ within the leaves and roots and representative images taken at different depths from the surface are available (Videos 1-6 and Fig. S7) and temporally confirm the above findings.

\section{Disease progress and plant growth}

As shown in Fig. 2 and S8-9, at 4 d, there was no impact on biomass as a function of treatment or disease. However, at 8 and $16 \mathrm{~d}$, disease reduced leaf biomass by $35.3 \%$ and $79.5 \%$ and root biomass by $20.1 \%$ and $73.5 \%$, respectively. The positive impacts of $S$ amendment were evident by day $8 ; \mathrm{cS}$ and bS significantly increased shoot biomass by $51.2 \%$ and $43.6 \%$, and root biomass by 26.7 and $48.2 \%$, respectively. By day 16 , the impact of bS became non-significant, but nS and cS increased shoot biomass by $264 \%$ and $378 \%$ and root biomass by $200 \%$ and $175 \%$, respectively.

Results from the $35 \mathrm{~d}$ greenhouse experiment were similar (Fig. S10 and S11). Treatment with the cS and nS decreased disease progress by 54 and $56 \%$, respectively; bS decreased disease by $32 \%$ but ionic $S$ had no impact on disease progression. Disease decreased plant shoot mass by $87.4 \%$ but $n S$ at 100 and 200 $\mathrm{mg} / \mathrm{L}$ increased shoot mass by $1,160 \%-1,750 \%$ relative to the disease controls; $\mathrm{cS}$ increases were $819 \%-1,180 \%$, respectively. Similarly, for the root mass, the increases were 263 and $412 \%$ for $n S$, and 219 and $221 \%$ for cS, respectively. Treatment with bS and iS had no impact on biomass under diseased conditions, regardless of concentration. Both nS and cS increased relative chlorophyll content compared

Page 11/31 
to the infested control and bS in the $16 \mathrm{~d}$ greenhouse experiment; in the $35 \mathrm{~d}$ greenhouse study, cS increased linear electron flow (LEF) relative to the infested control and bS, and had thicker leaves than bS (Fig. S12). This may be related to the increment of sucrose in corresponding leaves. cS increased leaf sucrose content by 1.4-1.5-fold over controls, nS, and bS at $8 \mathrm{~d}$; at $16 \mathrm{~d}$, both $\mathrm{nS}$ and cS had higher amount of sucrose than the infested control and bS by 50-68\%. Sucrose is the end product of photosynthesis and the primary sugar transported in the phloem of most plants. It also serves as both a source of carbon skeletons and energy for plant organs unable to perform photosynthesis.

A number of nanoscale amendments have been shown to reduce disease progression and increase biomass, although these have largely been with foliar applications. Elmer and White ${ }^{45}$ showed that foliar treatment of $\mathrm{CuO}$ significantly suppressed Fusarium infection and increased biomass of tomato and eggplant by $37.5 \%$ and $10.6 \%$, respectively, under field conditions. Similar fungal disease suppression capability has been reported for foliar application of different nanoscale forms of silica to watermelon ${ }^{46,47}$. Borgatta et al. ${ }^{16}$ and Ma et al. ${ }^{48}$ both demonstrated the importance of material properties, reporting that foliar application of $2 \mathrm{ml}$ of 10-1000 mg/: copper phosphate nanosheets suppressed Fusarium infection and increased the biomass of watermelon and soybean at concentrations as low as $10 \mathrm{mg} / \mathrm{L}$, whereas $>100 \mathrm{mg} / \mathrm{L}$ of spherical nanoscale $\mathrm{CuO}$ was needed to exert similar benefit. Less data is available on nanoscale sulfur amendments. Shang et al. ${ }^{49}$ reported that seed and foliar treatment of rice with CuS nanoparticles significantly suppressed Bakanae disease by 35.1-45.9\%. Importantly, results with the nanoscale materials were more effective than with Kocide (conventional fungicide). The current findings align with a previous study from our group that focused on seed treatment or foliar application of nanoscale $S$ in tomato ${ }^{50}$. Specifically, foliar application of $1 \mathrm{mg} / \mathrm{plant}$ significantly decreased disease by $47.6 \%$ and increased shoot biomass by $55.6 \%$. Importantly, the disease control efficacy was 3 -fold greater than hymexazol (conventional fungicide).

The mechanism of action for disease suppression clearly depends on the material used. Nanoscale $\mathrm{Cu}$ is known to uniquely stimulate plant defense systems and secondary metabolism ${ }^{48}$, although under sufficient concentrations, it can also directly act as an antimicrobial agent. Conversely, nanoscale silica can stimulate the deposition of cell water material in the roots, boosting the physical barrier protection against invading pathogens ${ }^{46,47}$. Nanoscale $\mathrm{S}$ is somewhat similar to $\mathrm{Cu}$; it is known to stimulate plant defense and the glutathione system, but also directly acts as an antimicrobial agent at sufficient doses 49 . For example, Cao et al. ${ }^{24}$ reported that the expression of select pathogenesis and oxidative stress genes were significantly upregulated by $11-352 \%$, although TEM confirmed the presence of nanoscale $S$ in the plant stem, potentially yielding direct antifungal effects.

\subsection{S accumulation}

In the 16-day experiment, disease had no impact on root $\mathrm{S}$ content at day 4 and 8 , but by harvest, the diseased root S content was $40 \%$ greater than the healthy controls (Fig. S13a). All S treatments increased 
the root S content at $16 \mathrm{~d}$ (range $25.2 \%-47.0 \%$ ), although the nanoscale $S$ treatments increased those levels more rapidly. Similar to the roots, the presence of disease significantly increased stem $S$ content in the untreated plants by day 16 (Fig S13b). Interestingly, all S treatments increased stem S content at $4 \mathrm{~d}$ (10.0\%-14.4\%), had no impact at $8 d$ and significantly reduced $S$ content at $16 \mathrm{~d}$ (range 11.9\%-24.8\%). Similar to the roots and stems, disease significantly increased leaf $S$ content at $4 \mathrm{~d}$ (Fig. 2c). All S treatments further increased leaf $S$ content $(6.1 \%-27.0 \%)$ at $4 \mathrm{~d}$; these effects were more marginal at $8 \mathrm{~d}$ but were again statistically significant at $16 \mathrm{~d}$.

The dynamics of tissue $S$ content as a function of time, disease and treatment are complex. It is clear that changes in tissue $\mathrm{S}$ content directly correlate with infection, often not evident at 4 or even $8 \mathrm{~d}$ but significantly greater by $16 \mathrm{~d}$. Even in the absence of treatment, this is a clear indication of increased $\mathrm{S}$ demand as a function of biotic stress and likely activation of secondary defense pathways that utilize S. Interestingly, S amendment did impact S tissue content, although this varied significantly with time, disease status, and treatment. In treated diseased plants, the impact of particle size and coating seemed to be minimal, suggesting that $S$ demand as a function of disease was the driving force for changes in the utilization pattern of this nutrient.

There are two known pathways for $\mathrm{S}$ assimilation in plants; the first involves $\mathrm{SO}_{4}{ }^{2-}$ by ion transporters SULTR, and the second with direct accumulation of elemental sulfur $\left(\mathrm{S}^{0}\right)$ catalyzed by Rhodaneses (Str), which are sulfotransferases ${ }^{51}$. Sulfate is the primary form assimilated by plants; however, most sulfur in soil exists in reduced forms. Under these circumstances, sulfur may become limiting and plants may increase the expression of sulfate transporters to facilitate acquisition by the roots. ST2.1 is expressed in xylem and phloem leaf parenchyma cells, xylem parenchyma and root pericycle cells $s^{52}$. ST2.1 mediates the uptake of sulfate from the apoplast within the vascular tissues in roots, and promotes translocation to young tissues through shoot phloem transport. Importantly, the sulfate pathway results in the formation of $\mathrm{SO}_{3}{ }^{2-}$ and $\mathrm{S}^{2-}$, both of which are harmful to plant cells. As shown in Fig. S19, the expression of ST2.1 with both nanoscale treatments is equivalent to the controls. In addition, as the first step of primary S-assimilation in plants, ATP-sulfurylase catalyzes the activation of sulfate $\left(\mathrm{SO}_{4}{ }^{2-}\right)$ and yields adenosine-5'-phosphosulfate (APS) that is reduced to sulfide $\left(\mathrm{S}^{2-}\right)$ and incorporated into cysteine (Cys) ${ }^{53}$. At $4 \mathrm{~d}$, ATP sulfurylase 1 (ATPSA1) expression was significantly downregulated with bS treatment in infected plants (Fig. 3b). No significant changes in nS and cS treatments occurred at any time points, indicating no $S$ deprivation and the $S$ uptake through this pathway was not significantly affected ${ }^{54}$. Conversely, at $8 \mathrm{~d}$ the expression of thiosulfate transferase (TST), which is a gene related to the elemental sulfur accumulation pathway, is significantly upregulated by both nanoscale treatments (Fig. 3a). Importantly, TST expression is unaffected by bS treatment. These findings indicate different pathways of sulfur accumulation and assimilation as a function of particle size. In plants, Str are found in the mitochondria and chloroplasts, as well as the cytoplasm and plastids. Niu et al. ${ }^{51}$ isolated a Str gene similar to AtStr1 from wheat that was resistant to the powdery mildew fungus Erysiphe graminis ${ }^{55}$. Similarly, Walz et al. ${ }^{56}$ isolated a rhodanese-like protein displaying similarity to AtStr17 from the phloem 
exudates of Curcubita maxima and reported its involvement in stress and defense response by acting as a phytohormone and/or in a signaling pathway.

\subsection{Tissue Nutritional Content}

The effect of S treatment on the uptake of select nutrients was also assessed (Fig. S14-16, S28-29, and Table S17). Stem accumulation of $\mathrm{Cu}, \mathrm{Fe}$ and $\mathrm{Zn}$ in the diseased plants were greater than in the healthy plants (Fig. S14). Treatment with S did not further influence stem content of these nutrients, except for Zn content with cS. Although stem Mn content was not affected by disease, exposure to the nS and cS significantly increased $\mathrm{Mn}$ concentration in this tissue. All S amendments, particularly cS, further increased leaf Fe content, whereas Ca content was significantly reduced with nS and cS (Fig. S15). Conversely, exposure to S did impact $\mathrm{Cu}$ and $\mathrm{Zn}$ leaf content. The translocation of $\mathrm{Mn}$ and $\mathrm{Si}$ was not affected by disease. However, under $S$ amendment, levels of these nutrients were increased, particularly with $\mathrm{nS}$ and cS. In roots (Fig. S16), cS significantly increased the Mn, Mg, and Fe at $4 \mathrm{~d}$ and $16 \mathrm{~d}$, and increased $\mathrm{Cu}$, and $\mathrm{P}$ at $16 \mathrm{~d}$ compared to the corresponding infested control, while no change was evident at $8 \mathrm{~d}$. No effects were noted with nS or bS at $16 \mathrm{~d}$. Less alteration was observed at $4 \mathrm{~d}$ or $8 \mathrm{~d}$ compared to $16 \mathrm{~d}$. Although nutrient level changes were evident with treatment, consistent trends were not evident, suggesting that the dynamics of these processes is complex and the impact on overall plant health is difficult to ascertain. The amount of bioavailable nutrient elements in soil was evaluated to examine whether the changes in root element accumulation were affected the $S$ based exposure (Fig. S17). In the DTPA extract, higher Zn content was found in cS treated soil than nS, bS, and the controls; cS resulted in higher soluble P content than bS in the same DTPA extract. However, such increments were not observed in the extracts by $\mathrm{DI}$ or $\mathrm{CaCl}_{2}$ solution. However, in general, the bioavailable nutrient element content in soil was only marginally affected by treatment.

\section{Gene expression}

Several genes related to the $S$ assimilation pathway were evaluated as a function of treatment and time (Fig. S19-20). In secondary $\mathrm{SO}_{4}{ }^{2-}$-assimilation, APS is phosphorylated in an adenylyl-sulfate reductase (ASR)-catalyzed reaction to produce 3'-phosphoadenosine 5'-phosphosulfate (PAPS). PAPS is involved in the production of other S-containing methionine-derived (aliphatic) or tryptophan-derived (indolic) secondary metabolites such as glucosinolates (GSs). GSs (particularly indolic type) have been reported to protect plants against several stressors and are required for plant immunity. In diseased plants, $\mathrm{nS}$ at $8 \mathrm{~d}$ and $\mathrm{CS}$ at $4 \mathrm{~d}$ and $8 \mathrm{~d}$ increased $A S R$ expression by $99.2 \%, 718.4 \%$, and $823.2 \%$ over the diseased control (Fig. S19). In addition, ASR expression at $4 \mathrm{~d}$ and $8 \mathrm{~d}$ in cS treated plants was 5.8- and 1.8-fold greater than bS treated plants. Glutathione (GSH) controls the redox states of many biomolecules, and functions in mediating enzymatic activity, xenobiotic detoxification, and influences plant growth, development, and stress management in response to both abiotic and biotic factors. Gamma-glutamylcysteine synthetase 
( $y G C S)$ catalyzes the first rate-limiting step in the production of GSH, involving the ATP-dependent condensation of cysteine and glutamate to form the dipeptide $y G C$. Here, disease increased $y G C S$ expression over time; expression at 8 and $16 \mathrm{~d}$ was $54.9 \%$ and $213.3 \%$ greater than levels at $4 \mathrm{~d}$. Treatment with $n S$ and $c S$ increased $y$ GCS expression at $4 \mathrm{~d}$ by $58.2-77.4 \%$ and $104.9-129.7 \%$ compared with untreated control and bS; interestingly, expression decreased at $16 \mathrm{~d}$ by $45.9-39.3 \%$ and $47.1-40.6 \%$. yGCS is known to be upregulated in plants under stress, including Fusarium infection, to form GSH and can be regarded as a biomarker of plant stress status ${ }^{57}$. From $4 \mathrm{~d}$ to $16 \mathrm{~d}$, the stress induced by Fusarium increased $\gamma G C S$ expression in the untreated diseased controls, but interestingly, at $16 \mathrm{~d}$, nS and cS reduced this response relative to bS and the controls. Similar results were evident for glutathione-S-transferases (GST), which are phase II detoxification enzymes that catalyze the conjugation of GSH to endogenous and exogenous electrophilic compounds. The presence of disease resulted in a time-dependent increase in GST expression. At $8 \mathrm{~d}$, cS significantly down-regulated GST expression relative to control and bS, indicating a partial alleviation of stress. These findings align with several transcriptome-wide investigations in the literature that have shown that the expression of specific GSTs is significantly increased during the early stages of pathogen infection.

Cysteine synthase (CS) produces cysteine, which is the primary product of sulfate assimilation, and also the reactant for methionine and glutathione synthesis. Cysteine is linked to the systemic acquired resistance pathway, which in turn activates a cascade of other resistance mechanisms to promote plant immunity from diseases ${ }^{58}$. Disease resulted in upregulation of CS at $8 \mathrm{~d}$ by $150.1 \%$ compared with the healthy control (Fig. S19). In addition, CS expression at $8 \mathrm{~d}$ was increased by $91.9 \%$ relative to $4 \mathrm{~d}$ by but by $16 \mathrm{~d}$, levels had decreased again. A similar trend was evident for the other treatments in diseased plants, indicating a time-dependent activation of CS with infection. $\mathrm{nS}$ and cS increased CS expression at most time points. At $16 \mathrm{~d}, \mathrm{nS}$ and $\mathrm{CS}$ induced CS expression levels that were $566.5 \%$ and $603.0 \%$ greater than bS. This particle size and time-dependent CS response is particularly interesting given the different pathways of $S$ accumulation noted above.

The ethylene pathway in plants is important in a range of developmental processes and is involved in signaling and response to stress. As shown in Fig. S20, in the first step of ethylene biosynthesis, SAMS2 catalyzes the reaction of ATP and methionine to form S-adenosylmethionine (SAM). Although disease did not cause any changes in SAMS2, cS resulted in a significant increase in SAMS2 expression (108.1\%) relative to untreated controls at $4 \mathrm{~d}$ by but not at 8 or $16 \mathrm{~d}$. ER69 is related to ethylene-responsive methionine synthase. At $8 \mathrm{~d}, \mathrm{nS}$ upregulated ER69 expression relative to bS and the disease control by $182.0 \%$ and $161.1 \%$, respectively; the increase in cS treatment compared with disease control at $8 \mathrm{~d}$ was 85.6\%. In addition, the ethylene-responsive factor (ERF) TSRF1 gene was monitored. ERFs are important in regulating pathogen resistance, abiotic stress tolerance and plant development. TSRF1 has been shown to be upregulated by ethylene, salicylic acid, and bacterial infection in tomatoes. Here, infection resulted in significantly greater expression at $8 d$ than at 4 or $16 d$. At $8 d$ and $16 d$, the highest level of expression was present in the control and bS, acting as a signal to fungal induced stress and clearly indicating that nanoscale treatments had alleviated the need for response ${ }^{17,48}$. The time-dependent 
nature by which nanoscale treatments inhibit disease progress is notable and supports previous findings suggesting a relatively narrow window of physiological opportunity where these crop protection strategies can be successful.

WRKY 6 codes for a plant resistance protein that provides specific immunity by recognizing F. oxysporum f. sp. lycopersici effectors ${ }^{59}$. This recognition results in effector-triggered immunity (ETI), a rapid plant defense response that inhibits successful infection. ETI occurs only between specific plant cultivars and pathogen strains based on the presence of corresponding R and Avr proteins. As shown in Fig. 3, at $8 \mathrm{~d}$, $\mathrm{nS}$ and cS significantly upregulated WRKY6 expression in infected tomato plants compared to control and bS by $280-365 \%$ and $212-282 \%$, respectively. Again, expression was significantly greater at $8 d$ than at 4 or $16 \mathrm{~d}$ (Fig. 3c).

A hierarchical clustering analysis was performed to differentiate the impacts of S-based materials on the expression of $S$ assimilation and disease-related genes in leaves harvested at 4, 8, and $16 \mathrm{~d}$. The metadata heatmap (Fig. S21a) shows the overall effect of exposure time and treatment on gene expression. A two-way ANOVA (Fig. S21b and Table S4) shows the effect of the two main factors, as well as their interaction effect, on the regulation of the 13 genes. The overall correlation heatmap and correlation analysis is shown in Fig. S22-23 and Table S5-7. CS was the gene with the greatest correlation with treatment; for exposure time, $T S T$ had the highest correlation. A correlation analysis performed with WRKL6 and $\gamma G C S$ revealed closely related expression to SRLK4 and ERF4, respectively.

To probe the effect of particle size and surface coating, the above analysis was performed within each $\mathrm{S}$ material group. The correlation heatmap (Fig. S24 and Table S8-10) displays different patterns for nS, cS, and bS. A Debiased Sparse Partial Correlation (DSPC) network analysis (Fig. 4 and Table S11-12) reveals the differential effect of the $S$ materials on the relationship between the analyzed genes. The edges represent the association measures between the two ends. Interestingly, in both nS and cS treatments, TST expression was strongly correlated with WRKL6; however, with bS, this relationship was much weaker. This highlights the importance of elemental $S$ uptake in nanoscale treatments yielding disease suppression. In addition, TST expression was correlated with TSRF1 and SAMS2 in both nS and cS treatments, but no such relationship was found for bS. These results indicate different mechanisms by which nS, cS, and bS effect plant metabolism and impact disease. A correlation analysis of the differentially affected relationship between all genes as a function of $S$ type is shown in Fig. S25. The PLS-DA analysis (Fig. S26 and Table S13) shows a clear separation of nS and cS, revealing a significant impact of exposure time on gene expression in these two treatments; this time-dependent effect is not evident in bS treatment.

An interesting time-dependent effect was evident after further data analysis at different time points. The correlation heatmap (Fig. S27 and Table S14-16) shows different patterns at $4 \mathrm{~d}, 8 \mathrm{~d}$, and $16 \mathrm{~d}$. The score plot obtained from PLS-DA (Fig. 5) shows that the separation between each group (nS, cS, bS, and control) was much clearer at $8 \mathrm{~d}$ than at $4 \mathrm{~d}$ or $16 \mathrm{~d}$. Importantly, according to the VIP score analysis (Fig. 5 and Table S18), the rank of TST among all the genes increased from the $9^{\text {th }}$ at $4 \mathrm{~d}$ to the $1^{\text {st }}$ at $8 \mathrm{~d}$, and 
then decreased to $6^{\text {th }}$ at $16 \mathrm{~d}$. Similarly, the VIP score of WRKY 6 increased from $8^{\text {th }}$ at $4 \mathrm{~d}$ to $3^{\text {rd }}$ at $8 \mathrm{~d}$, and then declined to $13^{\text {th }}$ at $16 \mathrm{~d}$. These data suggest a time sensitive physiological window whereby nanoscale $S$ treatments can significantly impact disease course ${ }^{17}$. A correlation analysis and the DSPC network of gene expression within each treatment was conducted at $4 \mathrm{~d}, 8 \mathrm{~d}$, and $16 \mathrm{~d}$, respectively (Fig. S30 and Table S19). Interestingly, the expression of TST gene starts to strongly correlate to WRKY6 at 8d, and that remains through $16 \mathrm{~d}$. This correlation does not occur at $4 \mathrm{~d}$. This result is consistent with the biomass data, which again support a nanoscale-specific mechanism of disease suppression through the enhanced elemental $\mathrm{S}$ uptake in $\mathrm{nS}$ and $\mathrm{cS}$ treatments.

\section{Metabolomics}

A total of 229 metabolites in tomato leaves were identified and semi-quantified. The relationship between all the metabolites and the enrichment analysis is shown in Fig. S31 and S32. The score plot obtained from the multivariate partial least-squares-discriminant analysis (PLS-DA) provides a general overview of the clustering information between groups and highlights good separation of $\mathrm{nS}$ and $\mathrm{cS}$ treatments from the bS and control groups at $16 \mathrm{~d}$ along the second principal component (PC2); however, bS and control are not well separated with each other (Fig. S33 and Table S20). There was no noticeable separation between the control, nS, cS, and bS groups at $8 \mathrm{~d}$. A clear separation was found within each $\mathrm{S}$ based treatment by $16 \mathrm{~d}$. This time-dependent response is in line with the gene expression and $\mathrm{S}$ accumulation data discussed above.

The heatmap (Fig. S35) shows the abundance of representative metabolites that affected the metabolic pathways related to disease responses. This systematic positive modulation of metabolic processes in tomato leaves upon $\mathrm{nS}$ and $\mathrm{cS}$ treatment suggests a generally beneficial impact on tomato metabolism. Specific metabolic pathways in the leaves were compared between $\mathrm{nS}$ and $\mathrm{bS}$ and also between $\mathrm{nS}$ and cS. At 8 d, nS enhanced 4 metabolic pathways and downregulated 3 pathways relative to bS (Fig. S36). Uridine monophosphate (UMP), L-glutamine, and sphinganine were the metabolites that were present at increased concentration with nS. The enhanced sphingolipid metabolism and aminoacyl-tRNA biosynthesis pathway was found at both $8 \mathrm{~d}$ and $16 \mathrm{~d}$ but the downregulation of anthocyanin biosynthesis, starch/sucrose metabolism, and flavone/flavonol biosynthesis was only evident at $8 \mathrm{~d}$. At $16 \mathrm{~d}, \mathrm{nS}$ resulted in significantly different metabolism of pyrimidine, glycerophospholipid, the folatederived single carbon pool, phenylalanine, phenylpropanoid biosynthesis, sphingolipid, lysine degradation, and aminoacyl-tRNA biosynthesis as compared to bS (Fig. S37). Specifically, nS significantly increased the level of tetrahydrofolate, sphinganine, and L-pipecolate compared to bS by $110.3 \%, 56.9 \%$, and $123.6 \%$, respectively, which likely enhanced the metabolic pathways of single carbon pool compounds (folate), aminoacyl-tRNA biosynthesis, sphingolipid, and lysine degradation. Conversely, the phenylalanine pathway was downregulated by nS compared to bS (by $119.0 \%$ ). Other important metabolites associated with the above affected pathways, such as choline phosphate and ferulate, were 
all greater with nS treatment than bS. Among the 8 affected pathways, 4 were enhanced and 1 was inhibited by nS as compared to bS.

The phenylpropanoid pathway results in the production of compounds responsive to pathogen infection and is a complex network regulated by multiple gene families that exhibit regulatory mechanisms that are involved in the production of anti-microbial compounds and signaling molecules. Manipulation of this pathway enhanced defensive systems, including salicylic acid and antimicrobial compounds ${ }^{60}$. Separately, sphingolipids are structural components of membranes and endomembrane systems and contribute to fluidity and other cellular functions, including defense against both abiotic and biotic stressors. The folate metabolic pathway influences a salicylic acid-independent interaction with plant immunity. Taken together, a nS-specific upregulation of these important metabolites is clearly indicative of enhanced plant defensive activity across a range of pathways and aligns well with the gene expression data.

In comparing pristine and coated nanoscale sulfur treated tomato leaves at $8 \mathrm{~d}$, cS enhanced 3 metabolic pathways and downregulated 3 pathways relative to nS (Fig. 38). D-glucose 6-phosphate, D-fructose 6phosphate, pyridoxal, and 12-oxophytodienoic acid were present at greater concentrations with cS; the decreased pathways included lower levels of UMP, L-glutamine, and L-proline with cS. Interestingly, the modulated regulation of these 6 pathways was observed only at 8d; these changes were not evident at 16 $\mathrm{d}$, highlighting a time and surface coating-dependent effect on cellular metabolism. At $16 \mathrm{~d}$, significant differences were found in 5 metabolic pathways (Fig. S39). Treatment with cS resulted in significant enhancement of isoquinoline alkaloid biosynthesis, tyrosine metabolism, and phenylalanine metabolism relative to nS; this was mainly due to the increased levels of tyramine and phenylacetaldehyde. Conversely, nS increased sphingolipid metabolism more than $\mathrm{cS}$, as evident by the increased amount of sphinganine by $56.9 \%$.

There were additional individual metabolites in tomato leaves that were uniquely modified by disease or treatment. nS increased proline, glutamine, and indole concentrations over control, $c S$, and bS by 1.6-2.0fold, 2.7-2.9-fold, and 1.4-2.2-fold, respectively. Proline not only protects plants from various stresses but also aids in recovery. Proline also plays pivotal roles in cell wall signal transduction cascades, plant development and stress tolerance ${ }^{61}$. In primary nitrogen assimilation, inorganic nitrogen is converted into glutamine and glutamate. Glutamine is a key signaling molecule and under stress conditions, participates in wound response, pathogen resistance, response and adaptation to abiotic stress, and long distance signal transduction ${ }^{62}$. Indole enhances the induction of defensive volatiles in neighboring plants in a species-specific manner ${ }^{63}$. Furthermore, the release of indole is essential for the priming of mono- and homoterpenes in plant leaves under attack. nS also increased methionine sulfoxide content by 1.6-fold relative to bS and controls. As noted above, methionine controls the level of key metabolites such as ethylene, polyamines, and biotin through SAM activity. Both biotic and abiotic stresses cause changes in methionine cycle (MTC) enzymes and these changes contribute to the ability of stress management ${ }^{64}$. 
cS increased p-aminobenzoic acid (pABA) (by 19-29\%) and pipecolic acid (by 48-56\%) over control, nS, and bS. pABA has been identified as an antifungal metabolite against a number of plant pathogens ${ }^{65}$ and it is known to trigger a systemic acquired resistance (SAR) against bacterial and viral pathogens ${ }^{66,67}$. Pipecolic acid, a non-proteinaceous product of lysine catabolism, is an important regulator of immunity in plants and accumulates upon infection, enhances resistance, and has been associated with systemic acquired resistance ${ }^{68,69} \mathrm{cS}$ also increased delphinidin content by $36 \%$ relative to bS, which is an anthocyanidin and an antioxidant. Fatty acids are associated in the early interactions between plants and pathogens, triggering a form of immunity that may help resist infection and colonization by pathogens ${ }^{70}$. cS increased palmitic acid by 1.4, 2.0 and 2.1-fold over the control, nS, and bS, and increased palmitic amide, a primary fatty acid amide derived from palmitic acid that has been reported to alleviate Fusarium disease in watermelon and tomato, by 1.7 -fold over bs ${ }^{71,72}$. Although jasmonic acid and methyl jasmonate were unaffected treatment, a significant increase in the precursor 12-oxo-Phytodienoic acid (12-OPDA) was induced by cS (2.9-fold) compared to controls. In addition to the link with jasmonic acid activity, 12-OPDA plays an independent role in signaling and mediating resistance to pathogens and pests. ${ }^{73}$ Accumulation of high 12-OPDA levels correlated with reduced ROS and elevated $\mathrm{GSH}^{74}$. Interestingly, no such increase was found with nS and bS.

L-tryptophan (LT), L-5-hydroxytryptophan (5-HTP; metabolite of tryptophan), and canthaxanthin, an effective antioxidant biosynthesized from $\beta$-carotene, were significantly up-regulated by $\mathrm{cS}(2.0,2.1$, and 1.7-fold, respectively) and $\mathrm{nS}(1.3,1.7$, and 2.2-fold, respectively) relative to bS. Tryptophan is an amino acid with an indole ring, and anchors membrane proteins within the cell membrane. Tryptophan is also important in glycan-protein interactions, alleviation of ROS damage, and functions as a biochemical precursor for niacin (vitamin $B_{3}$ ), melatonin and auxins. 3',5'-cyclic adenosine monophosphate (cAMP), a cyclic nucleotide, was increased by nS (2.3-fold), cS (2.7-fold), and bS (1.5-fold). cAMP is recognized as an important signaling molecule involved in sensing and response to biotic and abiotic environmental stresses for plant innate immunity, and potentially as a regulator of $\mathrm{Fe}$ and Ca homeostasis ${ }^{75}$. Tyrosine, an aromatic amino acid, was increased by $\mathrm{nS}$ and cS by 1.44 and 1.29 -fold compared to controls.

Tyrosine serves as a precursor of numerous specialized metabolites that have diverse physiological roles as electron carriers, antioxidants, attractants, and defense compounds ${ }^{76}$. Also, tyrosine-derived metabolites, such as tocopherols (vitamin E), plastoquinone, cyanogenic glycosides and suberin, have crucial roles in plant fitness. $\mathrm{nS}$ and $\mathrm{cS}$ also increased $p$-coumaric acid, a phenolic acid, by 1.5 and 1.2fold over bS and the controls. $p$-Coumaric acid exerts beneficial effects against several diseases due to its high free radical scavenging, pathogen suppression ${ }^{77}$, and antibacterial activities ${ }^{78}$.

\section{Rhizosphere microbiome analysis}

There were no significant differences in the number of amplicon sequence variants (ASVs) recovered between treatments, indicating minimal influence on the rhizosphere bacterial diversity, although the 
mean for the disease control was lower than the healthy or sulfur treated soils (Fig. S42). Similarly, the Shannon's diversity index returned no significant differences between the treatments, suggesting that the diversity of the community was generally resilient to the treatments. The taxonomic composition of the bacterial communities was also characterized at the phylum level (Fig. S42). Generally, the composition of the community, including the phyla Proteobacteria, Actinobacteriota, and Bacteroidota, was similar across all treatments. A PCA was performed (Fig. S42) which shows that the healthy controls clustered independently and significantly from the other groups (Table S21), suggesting the fungal pathogen exerted some effect on the rhizosphere community, independent of the form of sulfur added. Finally, a biomarker analysis was performed to identify if any bacterial taxa were enriched. Among the sulfur treatments, the enrichment of several taxa, particularly the Gammaproteobacteria and Proteobacteria, distinguished the bS treatment (Fig. S42), indicating that there were some taxa that differed in their response to the form of the sulfur amendments. Thus, taken together, these data point to small but measurable shifts in the tomato rhizosphere bacterial communities in response to the sulfur treatments, with some slight differences between the different sulfur types employed.

In conclusion, this study demonstrates that soil amended nS and cS significantly suppressed Fusarium disease in tomato plants as compared to bS. The time-dependent gene expression and metabolomic profile highlight $8 \mathrm{~d}$ as a critical period for NP-plant-pathogen interaction and disease suppression. Specifically, the expression of many defense and stress-related genes was significantly enhanced at 8d, whereas many of these alterations were reduced at $16 \mathrm{~d}$. Similarly, unique metabolomic profiles were evident $8 \mathrm{~d}$ and $16 \mathrm{~d}$ but not earlier. The potential mechanisms of $\mathrm{nS}$ and cS mediated disease suppression was investigated at the molecular level and was shown to be closely correlated with the enhanced expression of $\mathrm{S}$ bioassimilation and disease defense-related genes, increased disease resistance and plant immune system related metabolites, and more importantly, the unique $S$ assimilation pathway in which elemental $\mathrm{S}$ was directly transferred into plant tissue. This unique accumulation pathway allowed more efficient utilization of $\mathrm{S}^{0} \mathrm{NPs}$ by the plants and avoided the excessive formation of $\mathrm{SO}_{3}{ }^{2-}$ and $\mathrm{S}^{2-}$ which could be harmful to plant cells. This finding is supported by the upregulation of gene expression related to the element $S$ assimilation pathway in $\mathrm{nS} / \mathrm{cS}$ treatments, the increased $\mathrm{S}$ content in plant tissues and the unique $\mathrm{S}$ translocation ratio in $\mathrm{nS} / \mathrm{cS}$ treated samples relative to bS, as well as by two-photon microscopic images and videos. The findings demonstrate that soil application of $\mathrm{nS}$ and $\mathrm{cS}$ at an appropriate time offers great potential as a novel crop defense management strategy for disease suppression and significantly advances efforts to develop sustainable nanoscale treatments to achieve food security.

\section{Declarations}

\section{Acknowledgments-}

This work was supported by USDA-NIFA-AFRI 2020-67022-32416 and US FDA5U19FD007094-02. Jeremy L. Balsbaugh and Jen Liddle at Proteomics \& Metabolomics Facility at the University of Connecticut for 
technical assistance with the LC-MS/MS analysis.

\section{References}

1. Alexander, P. et al. Losses, inefficiencies and waste in the global food system. Agric. Syst. 153, 190200 (2017).

2. Willett, W. et al. Food in the Anthropocene: the EAT-Lancet Commission on healthy diets from sustainable food systems. Lancet 393, 447-492 (2019).

3. Dudley, N. \& Alexander, S. Agriculture and biodiversity: a review. Biodiversity 18, 45-49 (2017).

4. Urso, J. H. \& Gilbertson, L. M. Atom Conversion Efficiency: A New Sustainability Metric Applied to Nitrogen and Phosphorus Use in Agriculture. ACS Sustain. Chem. Eng. 6, 4453-4463 (2018).

5. Weithmann, N. et al. Organic fertilizer as a vehicle for the entry of microplastic into the environment. Sci. Adv. 4, eaap8060 (2018).

6. Wang, R. et al. A review of pesticide fate and transport simulation at watershed level using SWAT: Current status and research concerns. Sci. Total Environ. 669, 512-526 (2019).

7. Vollset, S. E. et al. Fertility, mortality, migration, and population scenarios for 195 countries and territories from 2017 to 2100: a forecasting analysis for the Global Burden of Disease Study. Lancet 396, 1285-1306 (2020).

8. Tripathi, A. D., Mishra, R., Maurya, K. K., Singh, R. B. \& Wilson, D. W. Estimates for World Population and Global Food Availability for Global Health. in The Role of Functional Food Security in Global Health 3-24 (Academic Press, 2019). doi:10.1016/B978-0-12-813148-0.00001-3.

9. Dhankher, O. P. \& Foyer, C. H. Climate resilient crops for improving global food security and safety. Plant. Cell Environ. 41, 877-884 (2018).

10. Kah, M., Tufenkji, N. \& White, J. C. Nano-enabled strategies to enhance crop nutrition and protection. Nat. Nanotechnol. 14, 532-540 (2019).

11. Iavicoli, I., Leso, V., Beezhold, D. H. \& Shvedova, A. A. Nanotechnology in agriculture: Opportunities, toxicological implications, and occupational risks. Toxicol. Appl. Pharmacol. 329, 96-111 (2017).

12. Giraldo, J. P., Wu, H., Newkirk, G. M. \& Kruss, S. Nanobiotechnology approaches for engineering smart plant sensors. Nat. Nanotechnol. 2019 146 14, 541-553 (2019).

13. Hofmann, T. et al. Technology readiness and overcoming barriers to sustainably implement nanotechnology-enabled plant agriculture. Nat. Food 1, 416-425 (2020).

14. Dimkpa, C. O. et al. Zinc oxide nanoparticles alleviate drought-induced alterations in sorghum performance, nutrient acquisition, and grain fortification. Sci. Total Environ. 688, 926-934 (2019).

15. Elmer, W. H. \& White, J. C. The use of metallic oxide nanoparticles to enhance growth of tomatoes and eggplants in disease infested soil or soilless medium. Environ. Sci. Nano 3, 1072-1079 (2016).

16. Borgatta, J. et al. Copper Based Nanomaterials Suppress Root Fungal Disease in Watermelon (Citrullus lanatus): Role of Particle Morphology, Composition and Dissolution Behavior. ACS Sustain. Chem. Eng. 6, 14847-14856 (2018). 
17. Shen, Y. et al. Copper Nanomaterial Morphology and Composition Control Foliar Transfer through the Cuticle and Mediate Resistance to Root Fungal Disease in Tomato (Solanum lycopersicum). J. Agric. Food Chem. 68, 11327-11338 (2020).

18. Ma, C. et al. Advanced material modulation of nutritional and phytohormone status alleviates damage from soybean sudden death syndrome. Nat. Nanotechnol. 15, 1033-1042 (2020).

19. Elmer, W. et al. Effect of Metalloid and Metal Oxide Nanoparticles on Fusarium Wilt of Watermelon. Plant Dis. 102, 1394-1401 (2018).

20. Bela, K. et al. Comprehensive analysis of antioxidant mechanisms in Arabidopsis glutathione peroxidase-like mutants under salt- and osmotic stress reveals organ-specific significance of the AtGPXL's activities. Environ. Exp. Bot. 150, 127-140 (2018).

21. Feinberg, A. et al. Reductions in the deposition of sulfur and selenium to agricultural soils pose risk of future nutrient deficiencies. Commun. Earth Environ. 2, 1-8 (2021).

22. Shankar, S., Pangeni, R., Park, J. W. \& Rhim, J. W. Preparation of sulfur nanoparticles and their antibacterial activity and cytotoxic effect. Mater. Sci. Eng. C 92, 508-517 (2018).

23. Roy Choudhury, S. et al. Surface-modified sulfur nanoparticles: An effective antifungal agent against Aspergillus niger and Fusarium oxysporum. Appl. Microbiol. Biotechnol. 90, 733-743 (2011).

24. Cao, X. et al. Elemental Sulfur Nanoparticles Enhance Disease Resistance in Tomatoes. ACS Nano (2021) doi:10.1021/ACSNANO.1C02917.

25. Gao, X., Spielman-Sun, E., Rodrigues, S. M., Casman, E. A. \& Lowry, G. V. Time and Nanoparticle Concentration Affect the Extractability of Cu from CuO NP-Amended Soil. Environ. Sci. Technol. 51, 2226-2234 (2017).

26. Adisa, I. O. et al. Role of Cerium Compounds in Fusarium Wilt Suppression and Growth Enhancement in Tomato (Solanum lycopersicum). J. Agric. Food Chem. 66, 5959-5970 (2018).

27. Ma, C. et al. Role of Nanoscale Hydroxyapatite in Disease Suppression of Fusarium -Infected Tomato. Environ. Sci. Technol. acs.est.1c00901 (2021) doi:10.1021/acs.est.1c00901.

28. Ma, C. et al. Time-Dependent Transcriptional Response of Tomato (Solanum lycopersicum L.) to $\mathrm{Cu}$ Nanoparticle Exposure upon Infection with Fusarium oxysporum f. sp. lycopersici. ACS Sustain. Chem. Eng. 7, 10064-10074 (2019).

29. Cota-Ruiz, K. et al. Copper nanowires as nanofertilizers for alfalfa plants: Understanding nano-bio systems interactions from microbial genomics, plant molecular responses and spectroscopic studies. Sci. Total Environ. 742, 140572 (2020).

30. Wang, Y. et al. Improvement of nutrient elements and allicin content in green onion (Allium fistulosum) plants exposed to CuO nanoparticles. Sci. Total Environ. 725, 138387 (2020).

31. Deng, C. et al. Bok choy (Brassica rapa) grown in copper oxide nanoparticles-amended soils exhibits toxicity in a phenotype-dependent manner: Translocation, biodistribution and nutritional disturbance. J. Hazard. Mater. 398, 122978 (2020). 
32. Acosta, Y. et al. Imaging cytosolic translocation of Mycobacteria with two-photon fluorescence resonance energy transfer microscopy. Biomed. Opt. Express 5, 3990 (2014).

33. Wang, Y. et al. Metabolomic analysis reveals dose-dependent alteration of maize (Zea mays L.) metabolites and mineral nutrient profiles upon exposure to zerovalent iron nanoparticles. Nanolmpact 23, 100336 (2021).

34. Xia, J. \& Wishart, D. S. Web-based inference of biological patterns, functions and pathways from metabolomic data using MetaboAnalyst. Nat. Protoc. 6, 743-760 (2011).

35. Pang, Z. et al. MetaboAnalyst 5.0: narrowing the gap between raw spectra and functional insights. Nucleic Acids Res. 49, W388-W396 (2021).

36. Chen, S. et al. $\mathrm{MoS}_{2}$ Nanosheets-Cyanobacteria Interaction: Reprogrammed Carbon and Nitrogen Metabolism. ACS Nano (2021) doi:10.1021/ACSNANO.1C05656.

37. Steven, B., LaReau, J. C., Taerum, S. J., Zuverza-Mena, N. \& Cowles, R. S. What's under the Christmas Tree? A Soil Sulfur Amendment Lowers Soil pH and Alters Fir Tree Rhizosphere Bacterial and Eukaryotic Communities, Their Interactions, and Functional Traits. Microbiol. Spectr. 9, (2021).

38. Alivisatos, A. P. et al. A unified initiative to harness Earth's microbiomes: Transition from description to causality and engineering. Science (80-). 350, 507-508 (2015).

39. Schloss, P. D. et al. Introducing mothur: Open-source, platform-independent, community-supported software for describing and comparing microbial communities. Appl. Environ. Microbiol. 75, 75377541 (2009).

40. Rognes, T., Flouri, T., Nichols, B., Quince, C. \& Mahé, F. VSEARCH: A versatile open source tool for metagenomics. PeerJ 2016, e2584 (2016).

41. Cole, J. R. et al. The Ribosomal Database Project (RDP-II): sequences and tools for high-throughput rRNA analysis. Nucleic Acids Res. 33, D294-D296 (2005).

42. Segata, N. et al. Metagenomic biomarker discovery and explanation. Genome Biol. 12, 1-18 (2011).

43. Villevieille, C. \& Novák, P. A metastable $\beta$-sulfur phase stabilized at room temperature during cycling of high efficiency carbon fibre-sulfur composites for Li-S batteries. J. Mater. Chem. A 1, 1308913092 (2013).

44. Fanxu Meng, D. King, M., A. Hassan, Y. \& M. Ugaz, V. Localized fluorescent complexation enables rapid monitoring of airborne nanoparticles. Environmental Science: Nano vol. 1 (Royal Society of Chemistry, 2014).

45. Elmer, W. H. \& White, J. C. The use of metallic oxide nanoparticles to enhance growth of tomatoes and eggplants in disease infested soil or soilless medium. Environ. Sci. Nano 3, 1072-1079 (2016).

46. Kang, H. et al. Silica nanoparticle dissolution rate controls the suppression of fusarium wilt of watermelon (citrullus lanatus). Environ. Sci. Technol. 55, 13513-13522 (2021).

47. Buchman, J. T. et al. Chitosan-Coated Mesoporous Silica Nanoparticle Treatment of Citrullus lanatus (Watermelon): Enhanced Fungal Disease Suppression and Modulated Expression of Stress-Related Genes. ACS Sustain. Chem. Eng. 7, 19649-19659 (2019). 
48. Ma, C. et al. Advanced material modulation of nutritional and phytohormone status alleviates damage from soybean sudden death syndrome. Nat. Nanotechnol. 15, 1033-1042 (2020).

49. Shang, H. et al. Copper sulfide nanoparticles suppress Gibberella fujikuroi infection in rice (Oryza sativa L.) by multiple mechanisms: contact-mortality, nutritional modulation and phytohormone regulation. Environmental Science: Nano vol. 7 (Royal Society of Chemistry, 2020).

50. Cao, X. et al. Elemental Sulfur Nanoparticles Enhance Disease Resistance in Tomatoes. ACS Nano (2021) doi:10.1021/ACSNANO.1C02917.

51. Papenbrock, J., Guretzki, S. \& Henne, M. Latest news about the sulfurtransferase protein family of higher plants. Amin. Acids 2010411 41, 43-57 (2010).

52. Maruyama-Nakashita, A. \& Ohkama-Ohtsu, N. Sulfur Assimilation and Glutathione Metabolism in Plants. in Glutathione in Plant Growth, Development, and Stress Tolerance 287-308 (Springer, Cham, 2017). doi:10.1007/978-3-319-66682-2_13.

53. Anjum, N. A. et al. ATP-sulfurylase, sulfur-compounds, and plant stress tolerance. Front. Plant Sci. 0, 210 (2015).

54. Prioretti, L., Gontero, B., Hell, R. \& Giordano, M. Diversity and regulation of ATP sulfurylase in photosynthetic organisms. Front. Plant Sci. 5, (2014).

55. Niu, J. S., Yu, L., Ma, Z. Q., Chen, P. Du \& Liu, D. J. Molecular cloning, characterization and mapping of a rhodanese like gene in wheat. Yi Chuan Xue Bao 29, 266-272 (2002).

56. Walz, C. et al. Proteomics of curcurbit phloem exudate reveals a network of defence proteins. Phytochemistry 65, 1795-1804 (2004).

57. Nawaz, F. et al. Sulfur-Mediated Physiological and Biochemical Alterations to Improve Abiotic Stress Tolerance in Food Crops. Plant Ecophysiol. Adapt. under Clim. Chang. Mech. Perspect. II Mech. Adapt. Stress Amelior. 415-441 (2020) doi:10.1007/978-981-15-2172-0_14.

58. Álvarez, C., Ángeles Bermúdez, M., Romero, L. C., Gotor, C. \& García, I. Cysteine homeostasis plays an essential role in plant immunity. New Phytol. 193, 165-177 (2012).

59. Catanzariti, A.-M., Lim, G. T. T. \& Jones, D. A. The tomato $1-3$ gene: a novel gene for resistance to Fusarium wilt disease. New Phytol. 207, 106-118 (2015).

60. Yadav, V. et al. Phenylpropanoid Pathway Engineering: An Emerging Approach towards Plant Defense. Pathog. 2020, Vol. 9, Page 312 9, 312 (2020).

61. Kishor, P. B. K., Hima Kumari, P., Sunita, M. S. L. \& Sreenivasulu, N. Role of proline in cell wall synthesis and plant development and its implications in plant ontogeny. Front. Plant Sci. 6, 1-17 (2015).

62. Qiu, X. M., Sun, Y. Y., Ye, X. Y. \& Li, Z. G. Signaling Role of Glutamate in Plants. Front. Plant Sci. 10, $1743(2020)$.

63. Erb, M. et al. Indole is an essential herbivore-induced volatile priming signal in maize. Nat. Commun. 6, 1-10 (2015). 
64. Mäkinen, K. \& De, S. The significance of methionine cycle enzymes in plant virus infections. Curr. Opin. Plant Biol. 50, 67-75 (2019).

65. Laborda, P., Zhao, Y., Ling, J., Hou, R. \& Liu, F. Production of Antifungal p-Aminobenzoic Acid in Lysobacter antibioticus OH13. J. Agric. Food Chem. 66, 630-636 (2018).

66. Trueman, C. L. \& Goodwin, P. H. Effects of para-aminobenzoic acid on bacterial speck symptom development and Pseudomonas syringae pv. tomato populations in tomato leaves. Eur. J. Plant Pathol. 160, 717-730 (2021).

67. Song, G. C., Choi, H. K. \& Ryu, C. M. The folate precursor para-aminobenzoic acid elicits induced resistance against Cucumber mosaic virus and Xanthomonas axonopodis. Ann. Bot. 111, 925-934 (2013).

68. Návarová, H., Bernsdorff, F., Döring, A. C. \& Zeier, J. Pipecolic Acid, an Endogenous Mediator of Defense Amplification and Priming, Is a Critical Regulator of Inducible Plant Immunity. Plant Cel/ 24, 5123-5141 (2013).

69. Bernsdorff, F. et al. Pipecolic Acid Orchestrates Plant Systemic Acquired Resistance and Defense Priming via Salicylic Acid-Dependent and -Independent Pathways. Plant Cel/ 28, 102-129 (2016).

70. Walley, J. W., Kliebenstein, D. J., Bostock, R. M. \& Dehesh, K. Fatty acids and early detection of pathogens. Curr. Opin. Plant Biol. 16, 520-526 (2013).

71. Liu, S. et al. Biological control of phytopathogenic fungi by fatty acids. Mycopathologia 166, 93-102 (2008).

72. Ma, K. et al. Palmitic acid mediated change of rhizosphere and alleviation of Fusarium wilt disease in watermelon. Saudi J. Biol. Sci. 28, 3616-3623 (2021).

73. Liu, W. \& Park, S. W. 12-oxo-Phytodienoic Acid: A Fuse and/or Switch of Plant Growth and Defense Responses? Front. Plant Sci. 12, 1687 (2021).

74. Sun, Y. H. et al. Accumulation of high OPDA level correlates with reduced ROS and elevated GSH benefiting white cell survival in variegated leaves. Sci. Rep. 7, 1-16 (2017).

75. Blanco, E., Fortunato, S., Viggiano, L. \& de Pinto, M. C. Cyclic AMP: A Polyhedral Signalling Molecule in Plants. Int. J. Mol. Sci. 21, 4862 (2020).

76. Schenck, C. A. \& Maeda, H. A. Tyrosine biosynthesis, metabolism, and catabolism in plants. Phytochemistry 149, 82-102 (2018).

77. Li, Y. et al. The plant phenolic compound p-coumaric acid represses gene expression in the Dickeya dadantii type III secretion system. Appl. Environ. Microbiol. 75, 1223-1228 (2009).

78. Lou, Z. et al. p-Coumaric acid kills bacteria through dual damage mechanisms. Food Control 25, 550-554 (2012).

\section{Figures}




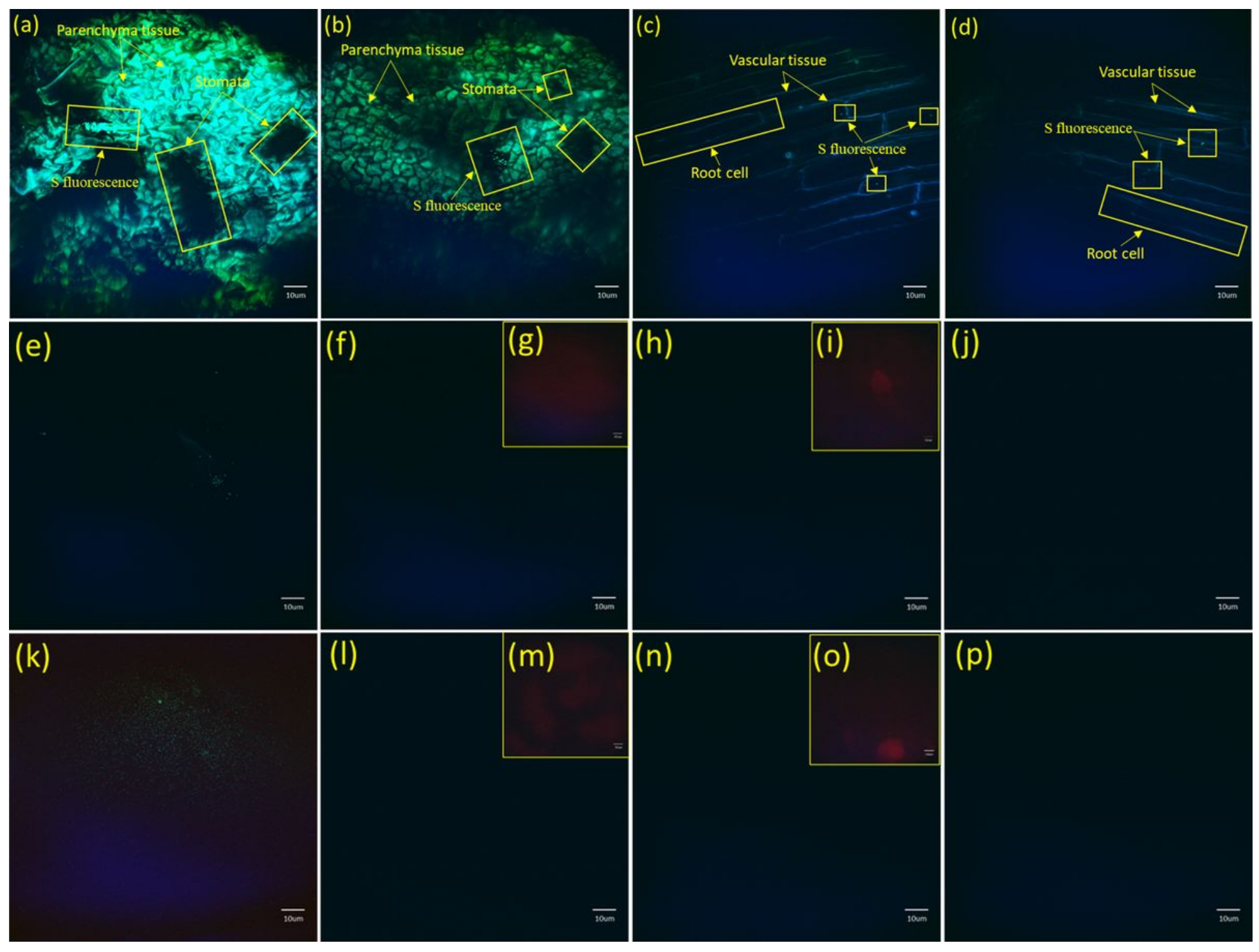

\section{Figure 1}

Two-photon microscope image of the characterization of pure $S$ based materials and Fusarium-infected tomato (leaf and root) upon exposure to nano S (nS), coated nano S (cS), and bulk S (bS) at $200 \mathrm{mg} / \mathrm{L}$ soil in first greenhouse study. Fluorescence signals are pointed out by arrows. Parenchyma, stomata, and vascular tissues are also shown. (a) Leaf of tomato treated by $\mathrm{nS}$ displaying fluorescence from $\mathrm{S}$ which are pointed out by arrows. Stomata and parenchyma tissues are also labeled. (b) Tomato leaf with $S$ fluorescence from bS treatment, which is specified by arrows in the square. The other arrows pointed out the structure of stomata and parenchyma tissues. (c) Root showing Cu fluorescence from nS treatment as indicated by arrows. The other arrows highlight the root vascular tissues. (d) Root exposed to cS. S fluorescence from cS exposure is pointed out by arrows. Other arrows describe the structure of root vascular tissues. Fig. (e) and (k) showed fluorescence signals from nS suspensions at $500 \mathrm{mg} / \mathrm{L}$ without or after sonication, showing weak or strong fluorescence, respectively. Fig. (f) and (i) shows cS suspensions at $500 \mathrm{mg} / \mathrm{L}$ without or after sonication, respectively. No fluorescent signal from $S$ is obtained. Fig. ( $\mathrm{g}$ ) and $(\mathrm{m})$ are $(\mathrm{f})$ and (i), respectively,with fluorescence signal received from a red channel (regarding the background) which show the existence of the non-luminous cS material. Fig. (h) and (n) 
display bS suspensions at $500 \mathrm{mg} / \mathrm{L}$ with or without sonication, respectively. Signal from $\mathrm{S}$ is not shown. The corresponding inserted pictures (i) and (o) correspond to Fig. (h) and (n) under a red-light mode, respectively, confirming the shape of the non-luminous bS material. Solutions of ionic $\mathrm{Na} 2 \mathrm{SO} 4$ without or with sonication are shown in Fig. (j) and (p), respectively. No representative fluorescent signal regarding $S$ was detected.
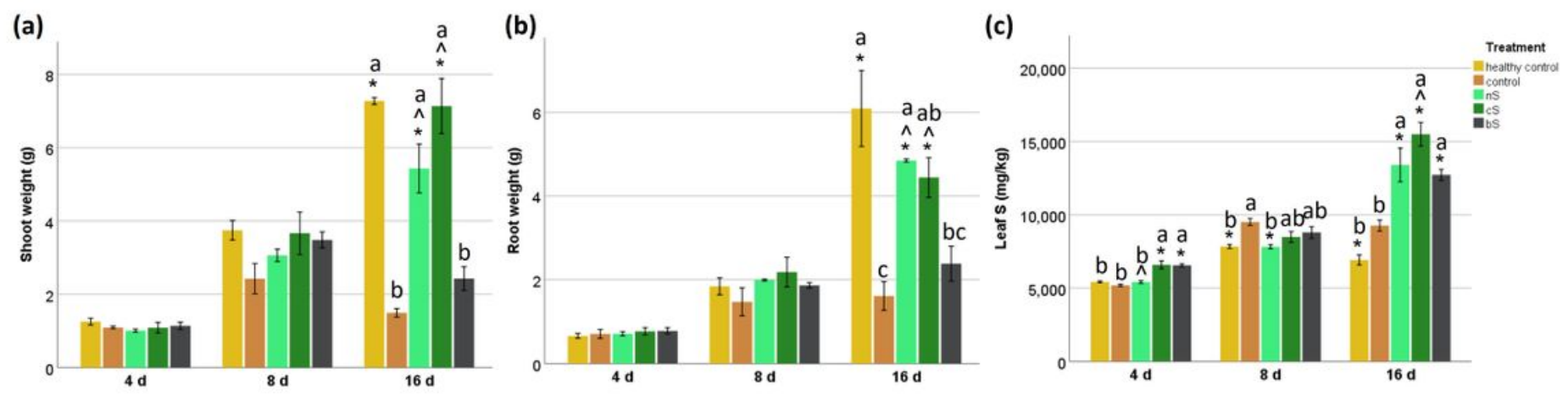

\section{Figure 2}

Physiological responses of Fusarium-infected tomato upon exposure to nano $\mathrm{S}(\mathrm{nS})$, coated nano $\mathrm{S}$ (cS), and bulk S (bS) at $200 \mathrm{mg} / \mathrm{L}$ soil in first greenhouse study with plants harvested at 4, 8, and 16 days after transplanting to infested media. Fig. represents (a) shoot fresh weight, (b) root fresh biomass, and (c) S concentration in leaves. In each panel, the yellow and orange bars represent the healthy control and diseased control, respectively; light and dark green bars represent the treatments with uncoated and stearic acid coated S NPs, respectively; and dark gray bars represent bulk S. Error bars correspond to the s.e.m. $(n=3)$. Values shown by different letters are significantly different at $p \leq 0.05$ (one-way ANOVA with a Tukey post hoc test). " $\star$ " represents significant difference between the diseased control and each $S$ treatment at $p \leq 0.05$ (Student t-test). " $\wedge^{\prime}$ indicates significant difference between bS and each S-based NPs treatment at $p \leq 0.05$ (Student t-test).
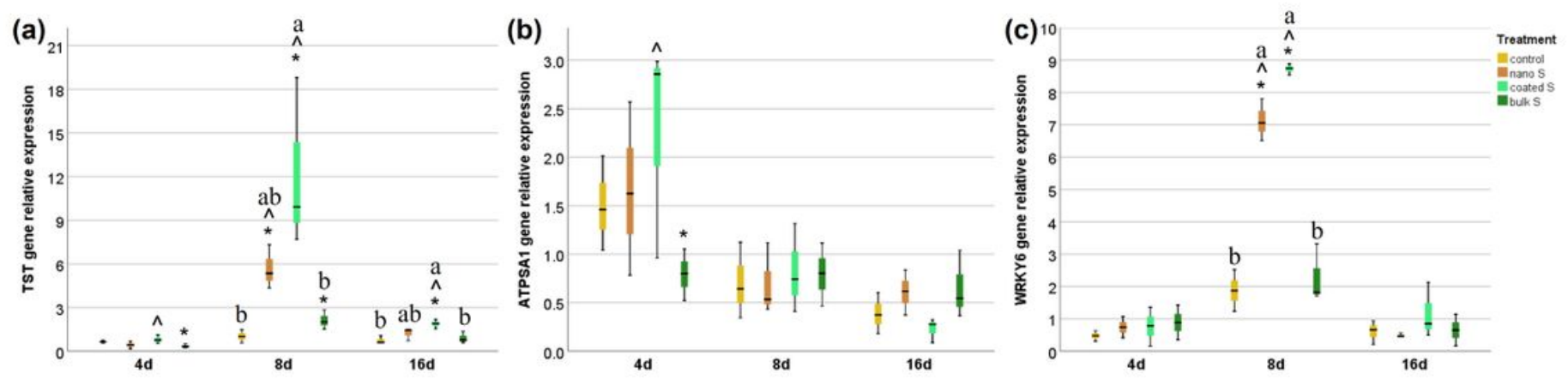

\section{Figure 3}

Relative expression of tomato $S$ bio-assimilation and disease resistance-related genes (TST, ATPSA1, WRKY6; a-c) in leaves of Fusarium-infected tomato upon soil exposure to nano $S$ (nS), coated nano $S$ (cS), and bulk S (bS) at $200 \mathrm{mg} / \mathrm{L}$ at $4 \mathrm{~d}, 8 \mathrm{~d}$, and $16 \mathrm{~d}$ after transplanting to infested media normalized to 
the healthy controls. Error bars correspond to the s.e.m. ( $n=9)$. Values shown by different letters are significantly different at $p \leq 0.05$ (one-way ANOVA with a Tukey post hoc test). " $\star$ " represents significant difference between the diseased control and each $S$ treatment at $p \leq 0.05$ (Student t-test). " ${ }^{\prime \prime}$ " indicates significant difference between bS and each S-based NPs treatment at $p \leq 0.05$ (Student t-test).
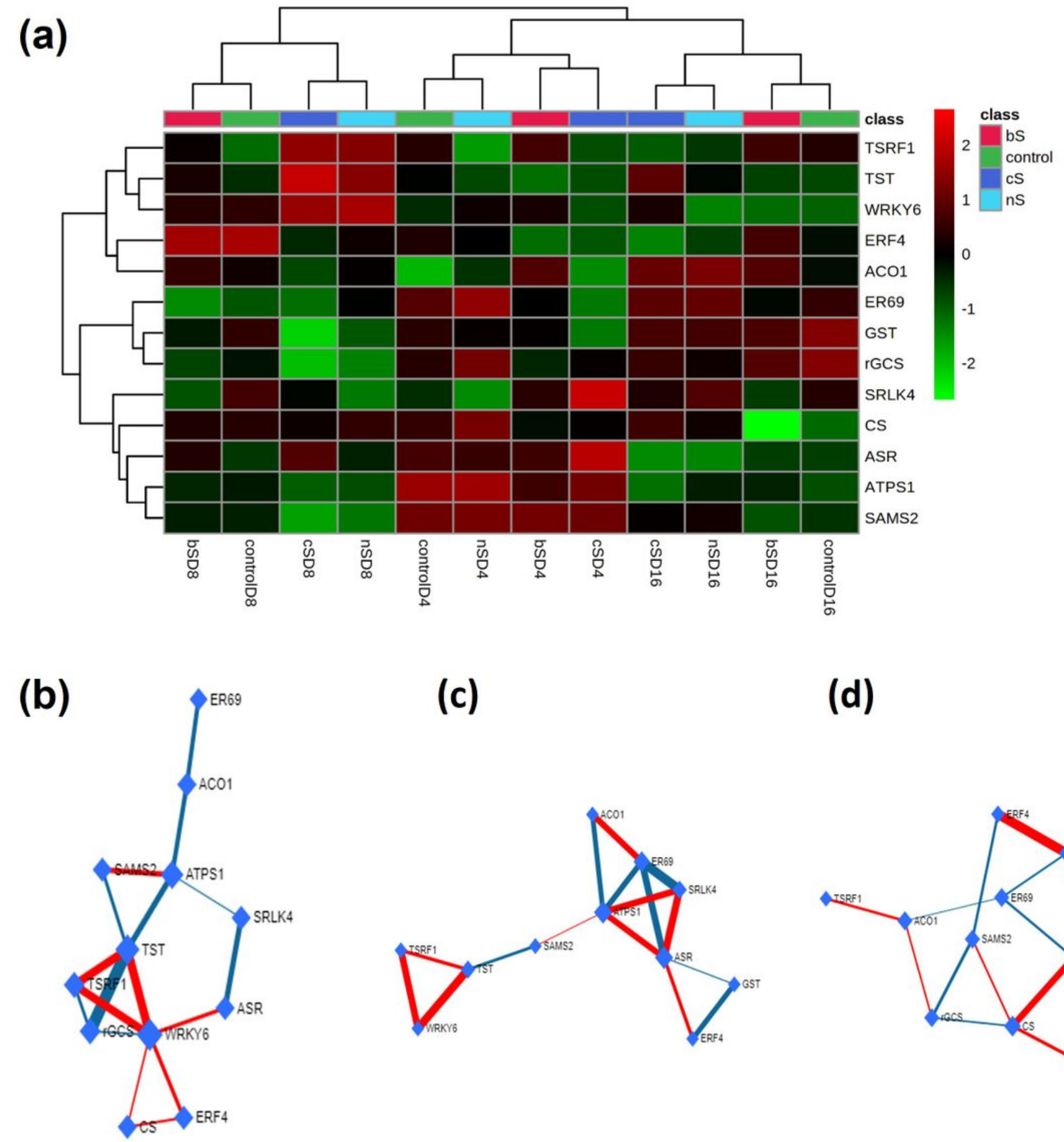

(c)

(d)
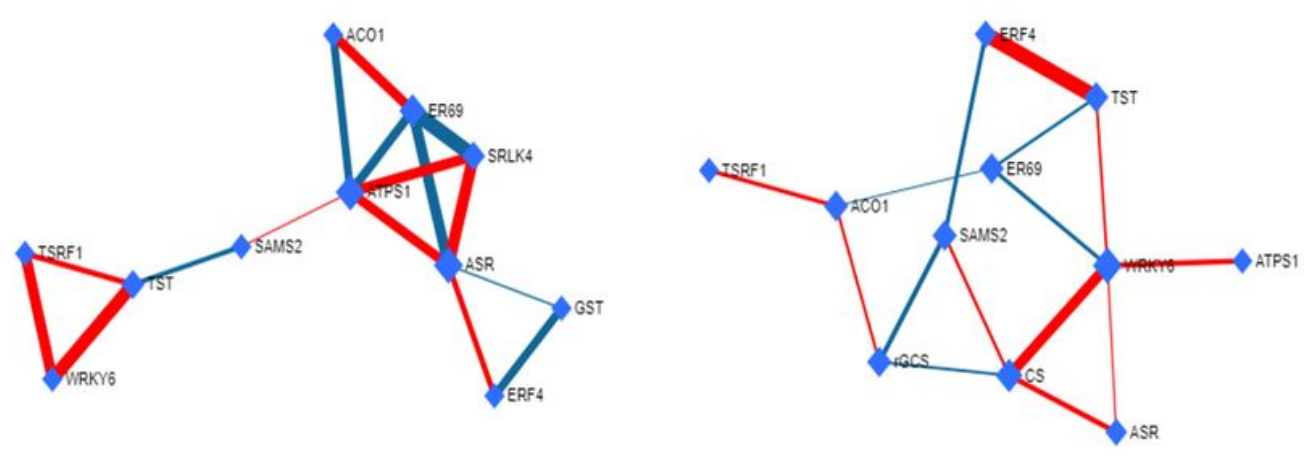

Figure 4

Hierarchical clustering heatmap and schematic diagram of schematic diagram from debiased sparse partial correlation (DSPC) network of tomato $S$ bio-assimilation and disease resistance-related genes. (a) Hierarchical clustering heatmap of the relative expression of all the targeted genes in Fusarium-infected 
tomato leaf upon exposure to nano S (nS), coated nano S (cS), and bulk S (bS) at $200 \mathrm{mg} / \mathrm{L}$ soil in first greenhouse study with plants harvested at 4, 8, and 16 days after transplanting to infested media. (b-d) Schematic diagram from debiased sparse partial correlation (DSPC) network analysis performed on the relative expression of all the targeted genes in Fusarium-infected tomato leaf of plants harvested at 4, 8, and 16 days after transplanting to infested media at $200 \mathrm{mg} / \mathrm{L}$ upon exposure to different $\mathrm{S}$ based materials as (b) nS, (c) cS, and (d) bS, respectively. Blue edges represent negative correlations while red edges represent positive correlations. The thicker the edge, the stronger the correlation between the features. Genes reported include TSRF1, TST, WRKY6, ERF4, AC01, ER69, GST, YGCS, SRLK4, CS, ASR, ATPS1, and SAMS2. The gene-fold changes were expressed relative to the healthy control plants.

(a)

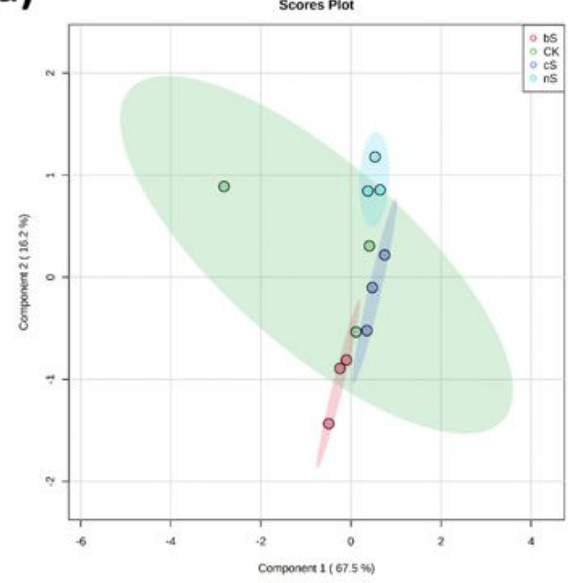

(d)

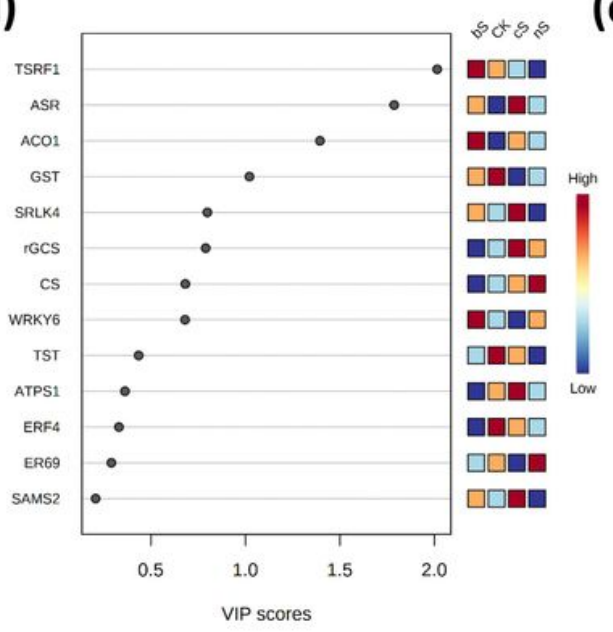

(b)

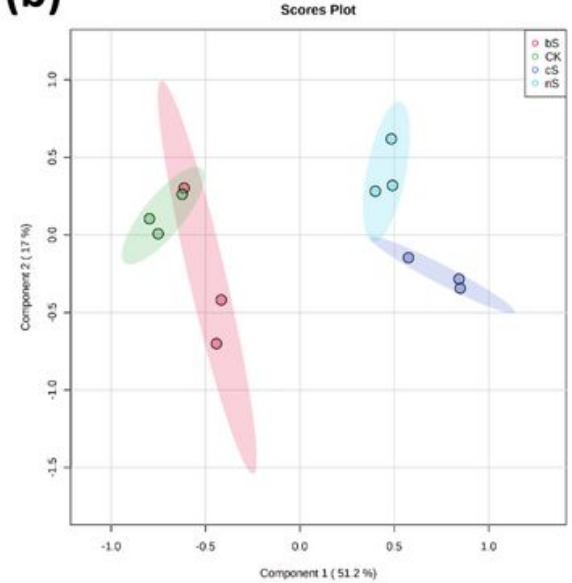

(e)

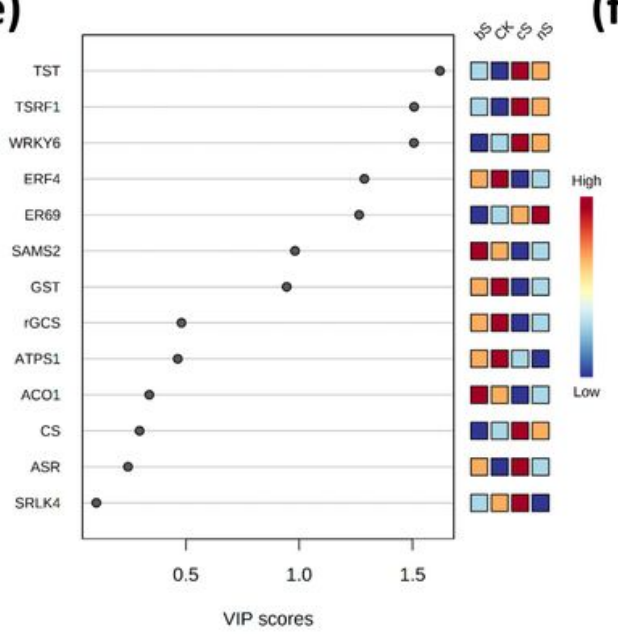

(c)
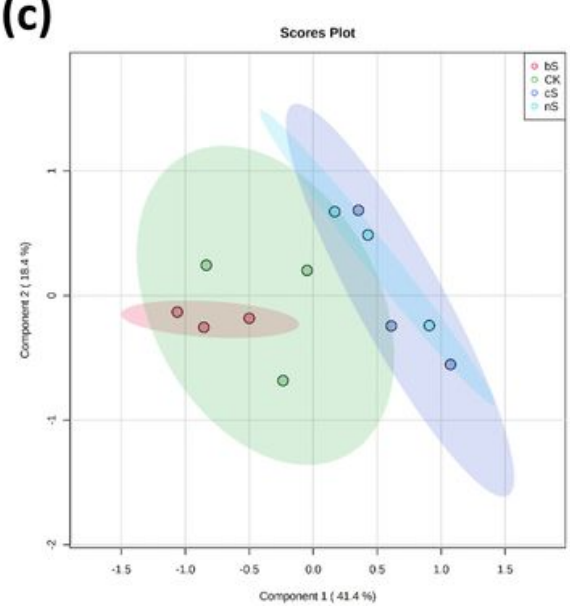

(f)

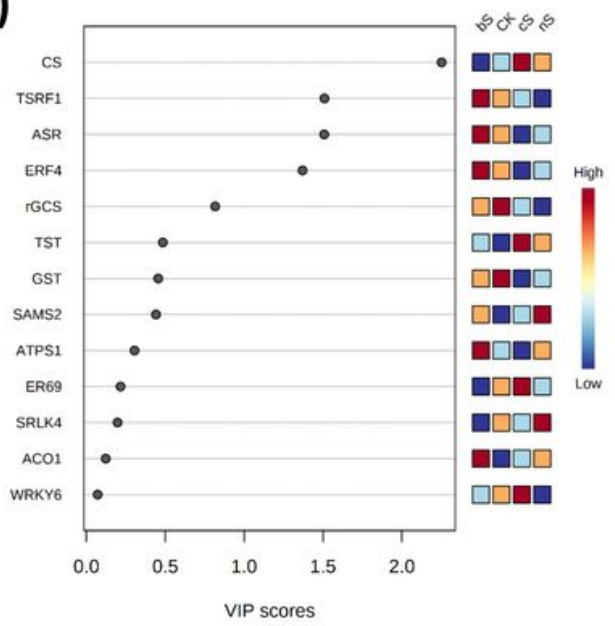

\section{Figure 5}

Multivariate analysis of the relative expression of all the targeted genes in Fusarium-infected tomato leaf upon exposure to nano S (nS), coated nano S (cS), and bulk S (bS) at $200 \mathrm{mg} / \mathrm{L}$ soil in the first greenhouse study harvested at 4, 8, and 16 days after transplanting to infested media. (a-c) Partial leastsquares discriminate analysis (PLS-DA) score plots of relative expression in leaves of tomato exposed to $S$ based materials (nS, cS, and bS) at different time point as (a) $4 \mathrm{~d}$, (b) $8 \mathrm{~d}$, and (c) $16 \mathrm{~d}$ after transplanting to infested media, respectively. (d-f) VIP score plot performed on the results of the relative gene expression at different time point as (d) $4 \mathrm{~d}$, (e) $8 \mathrm{~d}$, and (f) $16 \mathrm{~d}$ after transplanting to infested 
media, respectively. Genes reported include TSRF1, TST, WRKY6, ERF4, AC01, ER69, GST, yGCS, SRLK4, CS, ASR, ATPS1, and SAMS2. The gene-fold changes were expressed relative to the healthy control plants.

(a)
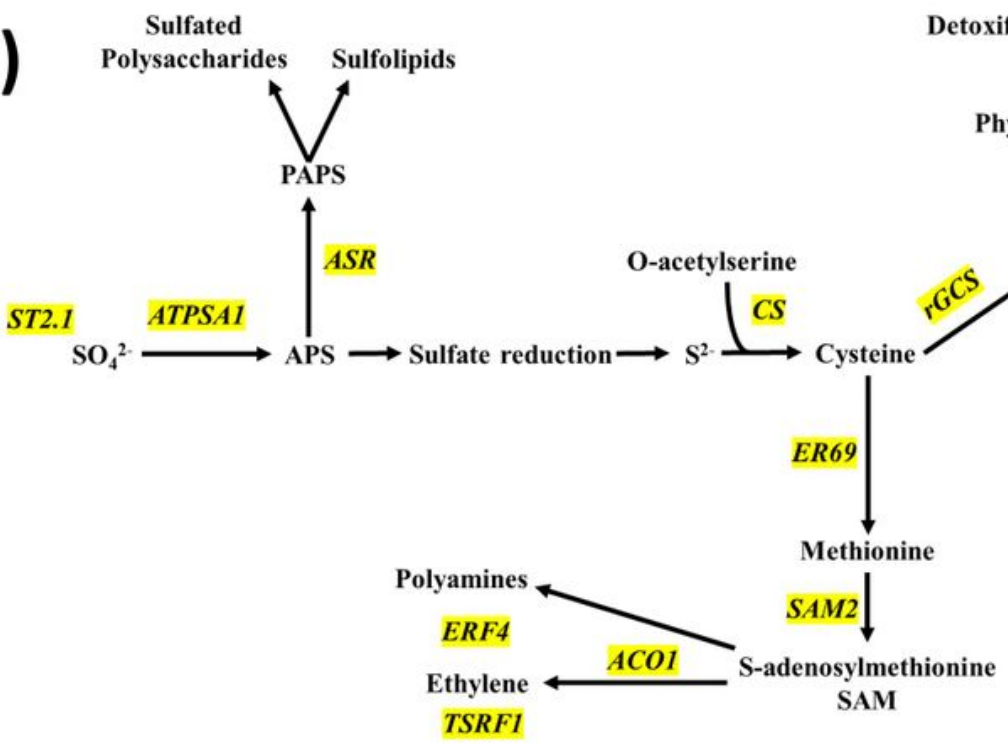

Detoxify heavy metals

Phytochelatins

Detoxify xenobiotics

GSH Disease resistance

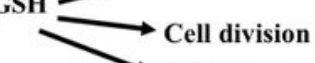

$\longrightarrow$ Cell division

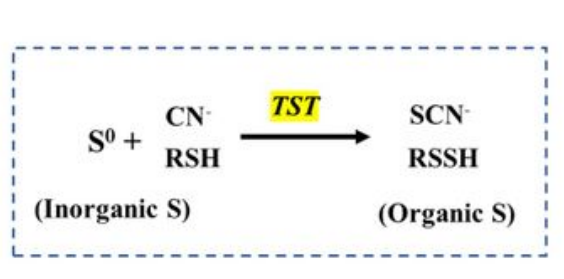

TSRFI

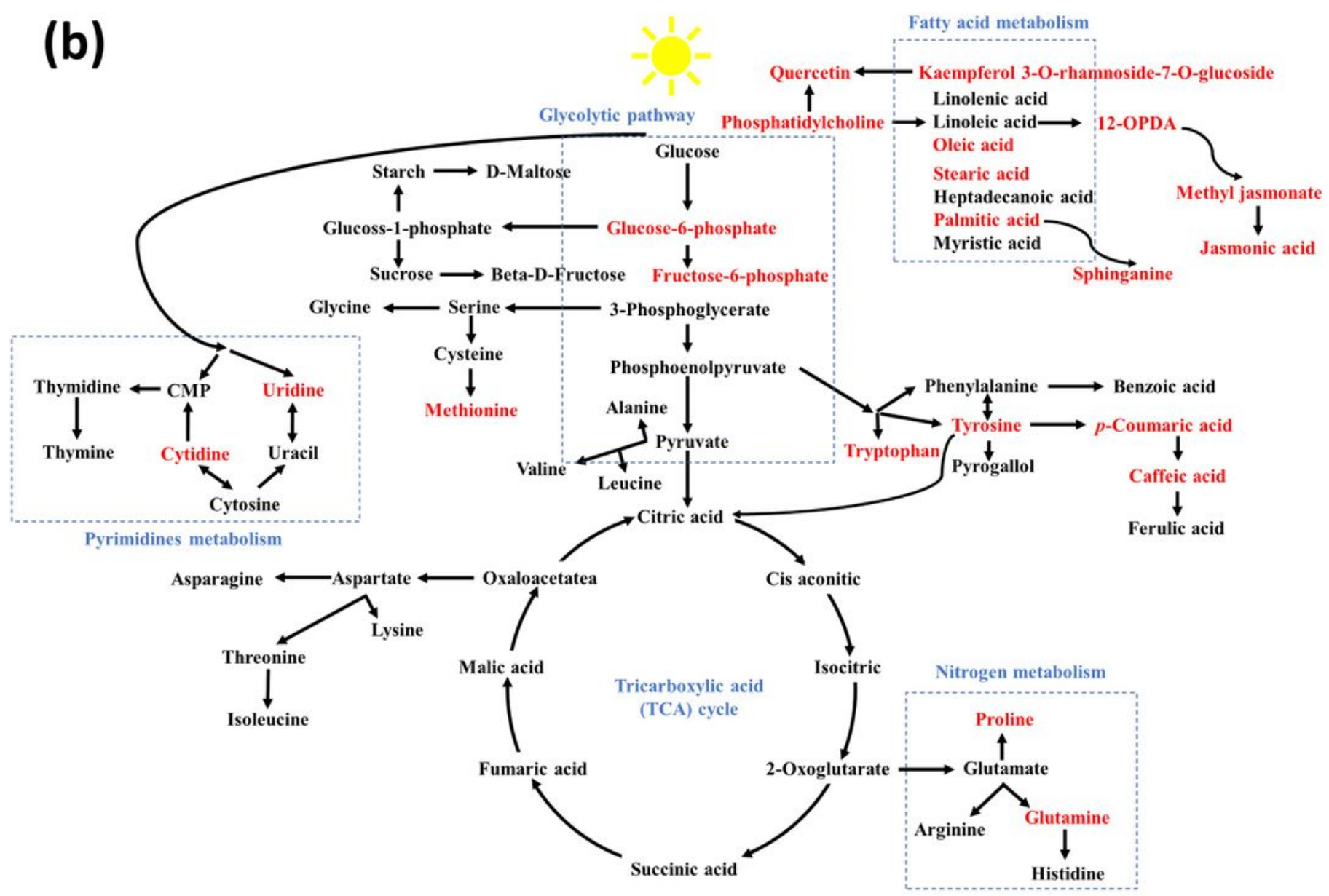

Figure 6

Schematic diagram of the targeted genes and representative metabolites in selected pathways. (a) Schematic diagram of targeted genes (marked using yellow color) that are related to the $S$ 
bioassimilation in tomato plants. (b) Schematic diagram of representative metabolites (labeled by red color) in Fusarium infested tomato leaf affected by the soil application of $S$ based material (nano $S$, coated nano S, and bulk S) at $200 \mathrm{mg} / \mathrm{L}$ soil at 8 and 16 days after transplanting to infested media. Arrow shows the direction of the reaction in the pathways.

\section{Supplementary Files}

This is a list of supplementary files associated with this preprint. Click to download.

- SIdataset.xlsx

- SITable.xlsx

- LeafnanoSvideo.avi

- LeafcoatedSvideo.avi

- LeafbulkSvideo.avi

- rootnanoSvideo.avi

- rootcoatedSvideo.avi

- rootbulkSvideo.avi

- SI.docx 